University of Nebraska - Lincoln

DigitalCommons@University of Nebraska - Lincoln

M. Eugene Rudd Publications

Research Papers in Physics and Astronomy

10-1985

\title{
Electron Production in Proton Collisions: Total Cross Sections
}

M. Eugene Rudd

University of Nebraska - Lincoln, erudd@unl.edu

Y-K. Kim

National Bureau of Standards, Gaithersburg, Maryland

D. H. Madison

Department of Physics, Drake University, Des Moines, lowa 50311

J. W. Gallagher

Joint Institute for Laboratory Astrophysics, University of Colorado and National Bureau of Standards,

Boulder, Colorado

Follow this and additional works at: https://digitalcommons.unl.edu/physicsrudd

Part of the Physics Commons

Rudd, M. Eugene; Kim, Y-K.; Madison, D. H.; and Gallagher, J. W., "Electron Production in Proton Collisions: Total Cross Sections" (1985). M. Eugene Rudd Publications. 43.

https://digitalcommons.unl.edu/physicsrudd/43

This Article is brought to you for free and open access by the Research Papers in Physics and Astronomy at DigitalCommons@University of Nebraska - Lincoln. It has been accepted for inclusion in M. Eugene Rudd Publications by an authorized administrator of DigitalCommons@University of Nebraska - Lincoln. 


\title{
Electron production in proton collisions: total cross sections
}

\author{
M. E. Rudd
}

Department of Physics and Astronomy, University of Nebraska-Lincoln, Lincoln, Nebraska 68588-0111

Y.-K. Kim

National Bureau of Standards, Gaithersburg, Maryland 20899

D. H. Madison

Department of Physics, Drake University, Des Moines, lowa 50311

\author{
J. W. Gallagher \\ Joint Institute for Laboratory Astrophysics, University of Colorado and National Bureau of Standards, \\ Boulder, Colorado 80309
}

Existing data on the ionization of neutral atoms and molecules by proton impact are reviewed, and electron production cross-section data are collected. The three major experimental methods are discussed and possible sources of error identified. Some theoretical cross sections are discussed, and well-established methods of relating them to measured cross sections are reviewed. A mathematical equation is fitted to the weighted experimental data for each target, and these fits are adjusted to be consistent with appropriate theoretical calculations and with electron impact and photoionization data. Recommended values of total cross sections for proton-impact ionization are given.

\section{GONTENTS}

I. Introduction

II. Definition of Cross Sections

III. Theoretical Considerations

A. Distorted-wave Born approximation

B. Relationship between proton- and electron-impact data

C. Comparison with photoionization data

D. Low-energy behavior

IV. Experimental Methods

A. Transverse-field method

1. General arrangement of apparatus

2. Preparation and collection of the proton beam

3. Determination of target gas density

4. Path length

5. Electron and ion collection

6. Spurious currents

a. Secondary electrons from slow ions striking the grid

b. Photoelectrons

c. Secondary electrons from surfaces struck by scattered beam particles

d. Sputtering and reflection of ions from the Faraday cup

7. Variations of the transverse-field method

B. Integration of differential cross sections

C. Energy-loss method

V. Survey and Critique of Existing Experimental Data

VI. Procedure for Choosing Recommended Values
A. Systematic errors
B. Weighting the data
C. Fitting equations
D. Recommended values
1. Atomic hydrogen
2. Helium

3. Neon

4. Argon

5. Krypton

6. Xenon

7. Molecular hydrogen

8. Nitrogen

9. Oxygen

10. Carbon monoxide

11. Ammonia

12. Carbon dioxide

13. Methane

14. Other targets

VII. Recommendations for Future Work Acknowledgments

References

\section{INTRODUCTION}

In the collision of an ion with a neutral atom or molecule, the atomic process that involves the largest transfer of energy is ionization or, more specifically, the ejection of an electron from the target. This process accounts for a large fraction of the energy loss of fast ions in materials. In such diverse fields as radiation damage and radiation biology, studies of the interaction of the solar wind with the upper atmosphere, magnetic and inertial confinement fusion studies, plasma physics, and stellar physics, data on the cross sections for ionization of various targets are necessary. However, even for the simplest case of proton collisions, there is no theoretical framework within which such cross sections can be reliably calculated for all impact energies and targets. Although theoretical treat- 
ments can supply some information for high energies and for a restricted number of targets, experiment must be relied upon to provide most of the data, as well as to provide a basis for further theoretical work.

There have been a few reviews of ionization measurement methods, e.g., Barnett and Gilbody (1968), and Massey and Gilbody (1974), and compilations of data (e.g., Barnett et al., 1977), but no comprehensive review of the cross sections. The review of Fedorenko (1959) was made at a time when only a small fraction of the presently available data had been measured.

Experimental data are presently available for many of the common gases. While the basic features of the dependence on proton energy are clear, there are wide discrepancies among the reported values of the cross sections. At low energies the spread among the measured cross sections is especially large, with factors of 2-4 common. Users of ionization data face the problem of choosing from among conflicting sets of data.

In this review we attempt to (1) make a comprehensive survey of existing experimental proton-impact ionization data available in the published literature, (2) evaluate each experiment for possible systematic errors, and (3) arrive at a set of recommended cross sections for each target, based on the best experimental and theoretical values available.

This study is restricted to data on gross or total electron production cross sections as defined in Sec. II. While the value of studies that give more detailed information, such as data on the ejection of electrons from inner shells, the production of various target charge states, or cross sections differential in the angle and energy of the ejected electrons is recognized, these are not considered here unless they yielded values for the total cross section for electron ejection. A separate review of differential cross sections for electron ejection to complement this study is planned.

In the next section definitions of the cross sections of interest in this review are given, and their relationship to the various measured cross sections is shown. In Sec. III the theoretical methods that can be used to calculate ionization cross sections or relate them to electron-impact and photoionization cross sections are briefly discussed. Section IV describes the three experimental methods used to measure cross sections and the possible sources of systematic errors. In Sec. $\mathrm{V}$ a list of the available experimental data and comments on the probable errors in each are given. Section VI describes the method used to arrive at a set of recommended values for each target and gives those values in the form of parameters of a fitting equation. Finally, some recommendations for further work in the subject are given.

The terms high, intermediate, and low energies denote the energy ranges where the projectile velocity is less than, approximately equal to, or greater than the velocity of the least tightly bound target electron, respectively. These regions correspond to the parts of the cross-section curve below, near, and above the maximum, respectively. Typically the maximum comes at an energy between 50 and $100 \mathrm{keV}$.

\section{DEFINITION OF CROSS SECTIONS}

When a proton collides with a neutral atom, electron production and capture processes may take place. The gross or total cross sections for production of electrons and resultant positive ions are defined as $\sigma_{-}$and $\sigma_{+}$, respectively. If $\sigma_{i e}$ is the cross section for producing $i$ electrons and $\sigma_{j c}$ is the cross section for capture of $j$ electrons by the incident proton, then

$$
\sigma_{+} \equiv \sum_{i} i \sigma_{i e}+\sum_{j} j \sigma_{j c}
$$

and

$$
\sigma_{-} \equiv \sum_{i} i \sigma_{i e}
$$

Note that $\sigma_{i e}$ includes all processes that produce $i$ electrons, including those in which other processes such as excitation or capture occur simultaneously. A similar remark holds for $\sigma_{j c}$. From these definitions it follows that

$$
\sigma_{+}-\sigma_{-}=\sum_{j} j \sigma_{j c} \text {. }
$$

For protons, only single and double capture are ever observed. Then

$$
\sigma_{+}-\sigma_{-}=\sigma_{1 c}+2 \sigma_{2 c} .
$$

Letting $\sigma_{c}$ stand for $\sigma_{1 c}$ and noting that the cross section for double capture is much smaller than that for single capture (see, for example, Williams, 1966), we have the approximation

$$
\sigma_{+}-\sigma_{-} \cong \sigma_{c}
$$

For other than monatomic targets, dissociation complicates the preceding analysis. If there are negatively charged dissociation products, then $\sigma_{-}$must be interpreted as the cross section for production of negative charge, i.e., negative ions plus electrons. If the cross section for electron production alone is required, $\sigma_{-}$must be reduced by the cross section for producing negative ions.

Some experimental data are available on the cross sections for producing slow positive ions of particular charge states $k$. If these cross sections are labeled $\sigma_{k+}$, then

$$
\sigma_{+}=\sum_{k} k \sigma_{k+}
$$

Another cross section that is sometimes measured is the "counting" cross section

$$
\sigma_{\text {count }}=\sum_{i} \sigma_{i e}
$$

In contrast to $\sigma_{-}$, which is usually obtained by a current measurement, $\sigma_{\text {count }}$ is obtained by counting events.

It will also be convenient to define the ratio

$$
\kappa_{-}=\frac{\sigma_{-}}{\sigma_{\text {count }}}=\frac{\Sigma i \sigma_{i e}}{\Sigma \sigma_{i e}} \text {. }
$$

Values of this quantity can be obtained from data on multiple ionization. 
In this review the primary emphasis will be on the cross section $\sigma_{-}$as defined by Eq. (2). In most measurements this quantity is obtained directly by a measurement of the electrons produced in the collision. In some it is derived from measurements of $\sigma_{+}$and $\sigma_{c}$ using Eq. (5). At high energies, $\sigma_{c}$ becomes negligible and then $\sigma_{-}=\sigma_{+} \cdot$ At least one group (Afrosimov et al., 1967) has measured cross sections for specific combinations of final projectile and slow-ion charge states, which can be combined to obtain $\sigma_{-}$. When data are available only on $\sigma_{+}$, they are included only if the energy is high enough so that $\sigma_{c}$ may be neglected. This is usually above about $250 \mathrm{keV}$.

\section{THEORETICAL CONSIDERATIONS}

Although no theoretical treatment is yet available that yields high-accuracy cross sections from threshold over the entire energy range of interest for all targets, there are important theoretical results that yield reliable asymptotic values for some cases, as well as relationships between cross sections for ionization by protons, electrons, and photons. In addition, progress has been made in improving the accuracy of approximations used in $a b$ initio quantal calculations.

Reviews of theoretical methods are available elsewhere (Massey and Burhop, 1969; Madison, 1973; Massey and Gilbody, 1974; Younger, 1985), so a comprehensive discussion of the theory of ionization will not be attempted here. The theoretical approach that has proved to be the most practical and reliable over a wide energy range is the distorted-wave Born approximation (DWBA). DWBA results have been used in this work in arriving at some of the recommended cross sections. The term DWBA, however, is generic and does not uniquely specify a particular calculation. In fact, many different calculations for a given process can all be appropriately labeled DWBA. In the next section the DWBA is developed to elucidate the types of choices that must be made in such a calculation, to discuss practical considerations not normally found in original research publications, and to point out the type of calculations that have been found to give the most reliable results.

\section{A. Distorted-wave Born approximation}

From a theoretical viewpoint, even the simplest ionization problems are difficult to treat in a satisfactory manner due to the three-body nature of the final state. It is convenient to consider the total ionization from beginning to end as an interaction between the three final-state particles-i.e., the incident projectile, the interacting electron, and the residual ion. In first-order perturbation theory, ionization may be viewed as a transition from an initial three-particle state to a final three-particle state caused by the interaction between the projectile and the atom. Theoretical approaches to ionization are usually formulated in terms of the descriptions of these three par- ticles. Classical methods typically assume that the incident projectile moves in some prescribed classical path and use quantum mechanics in the description of the active electron and/or ion. More rigorous treatments should use quantum mechanics for the description of all three particles. Even in a completely quantummechanical treatment, wave functions of varying accuracy may be used to represent the three different particles, depending on the scattering problem being considered. Here some of the relevant considerations that determine the necessary accuracy for the wave functions will be discussed.

For fast heavy projectiles, it is well known that inelastic scattering cross sections are dominated by events in which the projectile passes through the scattering region essentially undeflected. If experiments are being performed which do not determine the scattering angle of the projectile, but rather integrate over all projectile scattering angles, then important contributions will come only from very small angles of deflection (see, for instance, Park et al., 1978). For these cases, theoretical descriptions of the projectile such as quantum-mechanical plane waves or even classical straight lines are appropriate. If, on the other hand, angular distributions for the projectile were being measured, plane waves or straight lines could be expected to give reasonable results only for very small scattering angles. The assertion that plane waves are appropriate for the final-state description of the projectile may seem a bit peculiar, since the projectile is asymptotically in the Coulomb field of a separate electron and ion. The majority of the ionized electrons, however, have very low energies and leave the atom with a low velocity. Consequently, a fast-moving projectile will see an effectively neutral system during the time it is in the scattering region.

For the description of the interacting electron, a quantum-mechanical treatment must be used. Typical choices for the initial-state wave function for the interacting electron are either hydrogenic bound-state wave functions or more elaborate self-consistent field (SCF) wave functions such as Hartree-Slater or Hartree-Fock. For the final state, the active electron is in the Coulomb field of both the ion and the projectile. For slow-moving electrons, the projectile quickly leaves the scattering region and the dominant effect results from the residual ion. Since the active electron is always in at least the Coulomb field of the ion, plane-wave descriptions for the final state of the active electron are inappropriate. Typical choices for the final state of the active electron would include Coulomb waves for some effective charge or distorted waves for some effective potential chosen to represent the ion. The quality of the wave function needed to represent the active electron is also related to the experiment being considered. Experiments that yield detailed information about the active electron, such as energy and angular distributions, require more accurate descriptions for the active electron than do those experiments which integrate over some of the detailed information. Obviously, when an integration is performed, it is only necessary to model 
correctly the part that gives the largest contribution to the integral.

The ion is typically treated as a participant that is unchanged during the collision. The initial and final wave functions for the projectile and active electron are determined by the charge distribution of the ion. When independent-particle wave functions are used, theoretical considerations are greatly simplified by orthogonalizing the orbitals in the final-state wave functions to the corresponding orbitals in the initial-state wave functions. If this is not the case, the transition amplitudes contain many terms weighted by nonorthogonality factors (Madison and Merzbacher, 1975). A simple method for ensuring this orthogonality for the residual ion is to assume that its final state is the same as its initial state (the sudden approximation). In this approximation, the ionized electron is distorted by what may be called an initial-state ion. Even though this approach eliminates most of the additional terms in the transition amplitude which would appear for nonorthogonal wave functions, it does not necessarily eliminate all of them, since the continuum distorted wave is not necessarily orthogonal to the boundstate orbitals of the ion that created the distortion. The orthogonality would be automatic for initial and final hydrogenic wave functions of the same effective charge or SCF wave functions of the same localized potential, but not for Hartree-Fock wave functions.

The discussion up to this point has treated the projectile, active electron, and ion as essentially independent entities, without regard to the indistinguishability of identical particles. If the projectile is not an electron, the indistinguishability of the atomic electrons may be handled through the standard method of forming antisymmetric wave functions for the initial atom and final ion plus the ionized electron. For atomic shells that are full, the net result of such a process is simply to multiply the final results for distinguishable particles by the number of electrons in the shell. If the incident projectile is an electron, the process of antisymmetrization (of the projectile electrons and the atomic electrons) produces an additional exchange amplitude.

In this review, we are interested in integrated cross sections for proton-impact ionization. DWBA calculations (Madison, 1973; Manson et al., 1975; Rudd and Madison, 1976; Madison and Manson, 1979) have shown that reliable results for high-energy cross sections may be obtained by using the sudden approximation, by using plane waves for the initial- and final-state wave function for the proton, and by using distorted waves for the ejectedelectron wave functions. Consequently, we have obtained this type of DWBA results for use in determining recommended cross sections. There are still many different DWBA calculations of this type that can be performed by using different distorting potentials for the initial and final states of the active electron. Theoretical results quoted here use the Hartree-Fock wave functions of FroeseFischer (1972) for the initial state of the active electron. For the final state of the active electron, waves distorted by the initial-state ion (this ensures orthogonality for the inactive electron orbitals) are used. These distorted waves are obtained as follows. The initial-state Hartree-Fock orbitals for the neutral atom are used to form the final-state ion by removing the active electron orbital without modifying the remaining orbitals. This is a frozen-core approximation. A spherically averaged, local radial potential of the ion is then formed from the charge distribution of the ion represented by the remaining Hartree-Fock orbitals. The final-state distorted wave for the ionized electron is then calculated as an eigenfunction of this radial potential. These distorted waves are not necessarily orthogonal to the initial bound-state wave functions as is assumed in the theoretical development. For heavy atoms, it is important to ensure that the orthogonality requirement is satisfied. Here we have used an orthogonalization procedure of the Schmidt type, which will be described later.

It is important to note the conditions under which the theoretical calculations would be expected to be valid. The use of plane waves for the projectile is satisfactory as long as the proton is fast compared to the orbital velocity of the active electron, so that forward scattering is dominant. Plane waves would not be appropriate for largeangle differential cross sections for the proton. The present choice of final-state wave functions for the active electron should be satisfactory as long as the final-state interaction between the outgoing projectile and ejected electron is weak and the ionization event takes place fast enough so that the inactive electron orbitals do not relax before the active electron leaves. The latter condition is normally met for fast projectiles, but there are some important cases in which the former condition is not met. When the active electron is leaving the atom in the forward direction, with a speed comparable to that of the projectile, the final-state interaction between the active electron and projectile is not necessarily weak. If the projectile is a proton, ejected electrons are drawn toward the projectile, causing an enhanced cross section in the forward direction (Rudd and Macek, 1972). This process is called continuum electron capture (or charge transfer to the continuum), and for some circumstances the enhanced cross sections are so large that use of theoretical methods that do not include this effect results in a substantial underestimation of the ionization cross section. To include this effect in a perturbation approach, second-order terms must be calculated, but that is beyond the scope of this study. Salin (1972) has obtained a first-order correction factor for DWBA ejected electron angular distributions designed to account for continuum electron capture.

In the distorted-wave Born approximation, the triple differential cross section (differential in momentum transferred by the projectile and in energy and angle of the ejected electron) for ionization of an atomic shell containing $N$ electrons is given in atomic units by (Madison, 1973)

$$
d \sigma_{f i}=4 \pi N z^{2} v^{-2}\left|F_{f i}\left(\mathbf{q}, E_{e}, \Omega_{e}\right)\right|^{2} q^{-2} d\left(\ln q^{2}\right) d E_{e} d \Omega_{e},
$$


where $z$ is the projectile charge, $v$ is the relative projectile-atom velocity before the collision, $E_{e}$ is the ejected electron energy, and $\Omega_{e}$ is the solid angle of observations for the ejected electron. The momentum transfer $q$ is defined by

$$
\hbar^{2} q^{2}=p^{2}+p^{\prime 2}-2 p p^{\prime} \cos \theta_{p},
$$

where $p$ and $p^{\prime}$ are the initial and final momenta of the projectile and $\theta_{p}$ is the scattering angle of the projectile. Equation (9) can be converted into the angular distribution of the projectile through Eq. (10). For wave functions formed from single-particle orbitals, the form factor $F_{f i}$ is given by

$$
F_{f i}=\left\langle\chi_{f}^{(-)}(\mathbf{k}, \mathbf{r})\left|e^{i q \cdot \mathbf{r}}\right| \psi_{i}(\mathbf{r})\right\rangle,
$$

where $\chi_{f}^{(-)}$is the incoming final-state, energy-normalized distorted wave for the active electron of wave number $\mathbf{k}(=\mathbf{p} / \hbar)$. Here $\psi_{i}$ is the initial bound-state orbital. For an atom, this orbital is written as

$$
\psi_{i}(\mathbf{r})=r^{-1} \psi_{n l_{0}}(r) Y_{l_{0} m_{0}}(\Omega),
$$

where $n l_{0} m_{0}$ are the bound-state quantum numbers. The energy-normalized (per Rydberg) final-state distorted wave may be expressed as

$\chi_{f}^{(-)}(\mathbf{k}, \mathbf{r})=(k \pi)^{-1 / 2} r^{-1} \sum_{l m} i^{l} \chi_{l}(k, r) Y_{l m}\left(\Omega_{e}\right) Y_{l m}^{*}(\Omega)$.

The radial part of the distorted wave satisfies the following equation:

$$
\left[\frac{d^{2}}{d r^{2}}-\frac{l(l+1)}{r^{2}}-V(r)+k^{2}\right] \chi_{l}(k, r)=0,
$$

where $V(r)$ is the spherically averaged initial-state ion potential discussed previously. The boundary conditions for an ejected electron in a Coulomb field require that asymptotically the radial function has the following form:

$\chi_{l}(k, r) \underset{r \rightarrow \infty}{=} e^{i\left(\sigma_{l}+\delta_{l}\right)} \sin \left[k r-k^{-1} \ln (2 k r)-\frac{1}{2} l \pi+\sigma_{l}+\delta_{l}\right]$.

Here $\sigma_{l}$ is the Coulomb phase shift and $\delta_{l}$ is the additional phase shift resulting from the non-Coulomb part of the distorted potential. To obtain the single differential cross section (differential in energy of the ejected electron) of interest here, Eqs. (12) and (13) are inserted in Eq. (11) and the exponential is expanded in terms of spherical Bessel functions. Then the results are used in Eq. (9) and integrated over $\Omega_{e}$ and $q$. The single differential cross section for unpolarized protons incident upon an unpolarized target is given by

$$
\frac{d \sigma_{f i}}{d\left(E_{e} / R\right)}=\frac{4 \pi N z^{2}}{k v^{2}} \sum_{l \lambda}(2 \lambda+1) C\left(l_{0} \lambda l ; 000\right)^{2}\left|A_{l \lambda l_{0}}^{k}\right|^{2},
$$

where $C$ is a Clebsch-Gordan coefficient, $R$ is the Ryd- berg energy, and

$$
A_{l \lambda l_{0}}^{k}=\int_{q_{\min }}^{q_{\max }} M_{l \lambda l_{0}}^{k q} q^{-2} d\left(\ln q^{2}\right)
$$

and

$$
M_{l \lambda l_{0}}^{k q}=\left\langle r^{-1} \chi_{l}(k, r)\left|j_{\lambda}(q r)\right| r^{-1} \psi_{n l_{0}}(r)\right\rangle,
$$

where $j_{\lambda}(q r)$ is a spherical Bessel function. To obtain the total cross section (dependent only on the energy of the incident projectile), the single differential cross section [Eq. (16)] must be integrated over the energy of the ejected electron. This total cross section is $\sigma_{1 e}$ defined in Sec. II.

In summary, a series of steps is required to obtain theoretical distorted-wave Born cross sections for ionization. As a concrete example, the required steps for ionization of neon are considered. Neon has three subshells from which an electron can be ejected. Since, in the experiments, there is no distinction between the various subshells, cross sections for each subshell must be independently calculated. The first step in the process is to obtain the Hartree-Fock wave functions $\psi_{n l_{0} m_{0}}$ for each of the three subshells. Next, these wave functions are used to calculate three different initial-state ionic potentials $V(r)$ corresponding to the electronic configurations $1 s^{1} 2 s^{2} 2 p^{6}, 1 s^{2} 2 s^{1} 2 p^{6}$, and $1 s^{2} 2 s^{2} 2 p^{5}$. Then the single differential (energy-dependent) cross section for each of the subshells is calculated. This is accomplished by (a) using the appropriate ionic potential in Eq. (14) to obtain distorted waves for various angular momenta (here $l \leq 15$ was used), (b) numerically evaluating the appropriate integrals [Eqs. (17) and (18)], and (c) obtaining the cross section Eq. (16), for a particular ejected-electron energy. This process must be repeated for various electron energies on a mesh suitable for integration to obtain the total cross section. Since the cross sections change more rapidly for slow ejected electrons than for fast ones, a finer mesh is used for slow electrons. Here cross sections for ejected-electron energies $E_{e}=2-500 \mathrm{eV}$ were calculated. Numerical instabilities restrict the lowest calculated ejected-electron energy to about $0.1 \mathrm{eV}$. Since the integral over ejected-electron energies starts at $E_{e}=0$, the single differential cross sections must be extrapolated to zero kinetic energy. The results are not very sensitive to the extrapolation algorithm. The integral over ejected-electron energy is then performed for each of the subshells and the subshell results are summed to obtain the total ionization cross section,

$$
\sigma_{\text {ion }}=\sum_{j} \int\left(d \sigma_{j} / d E_{e}\right) d E_{e},
$$

where the summation is for all atomic subshells.

In performing calculations such as this, there are several numerical parameters that must be constantly monitored, such as the maximum number of $l$ values required in the partial-wave expansion of the distorted waves. In general, the necessary number of partial waves increases with increasing energy of the ejected electron. In general, ten $l$ values are sufficient for slow ejected elec- 
TABLE I. Comparison of distorted-wave Born and recommended cross sections (in $10^{-20} \mathrm{~m}^{2}$ ).

\begin{tabular}{|c|c|c|c|c|c|c|c|c|}
\hline \multirow{2}{*}{$\begin{array}{l}\text { Atom } \\
\text { Proton energy } \\
(\mathrm{MeV})\end{array}$} & \multicolumn{2}{|c|}{$\mathrm{H}$} & \multicolumn{2}{|c|}{$\mathrm{He}$} & \multicolumn{2}{|c|}{$\mathrm{Ne}$} & \multicolumn{2}{|c|}{$\mathrm{Ar}$} \\
\hline & 0.25 & 5.0 & 0.25 & 5.0 & 0.25 & 5.0 & 0.25 & 5.0 \\
\hline \multicolumn{9}{|l|}{ Subshell } \\
\hline $1 s$ & 0.640 & 0.048 & 0.605 & 0.057 & 0.00024 & 0.00064 & $1.5 \times 10^{-6}$ & $6.7 \times 10^{-5}$ \\
\hline $2 s$ & & & & & 0.148 & 0.013 & 0.0053 & 0.0020 \\
\hline $2 p$ & & & & & 1.31 & 0.162 & 0.024 & 0.013 \\
\hline $3 s$ & & & & & & & 0.437 & 0.026 \\
\hline $3 p$ & & & & & & & 4.38 & 0.437 \\
\hline Total $^{\mathrm{a}}$ & 0.640 & 0.048 & 0.605 & 0.057 & 1.76 & 0.176 & 4.85 & 0.479 \\
\hline $\begin{array}{l}\text { Recommended } \\
\text { values }^{\text {b }}\end{array}$ & 0.625 & 0.047 & 0.615 & 0.056 & 1.47 & 0.165 & 3.66 & 0.394 \\
\hline
\end{tabular}

${ }^{a}$ The theoretical cross sections are $\sigma_{\text {count }}$ defined by Eq. (7). Therefore these values should be lower than the recommended values listed below, which are values of $\sigma_{-}$defined by Eq. (2).

bThese values were obtained using Eq. (31) and the parameters in Table II.

trons $(E<100 \mathrm{eV}$ ), while more $l$ values are required for $100<E \leq 500 \mathrm{eV}(\sim 15)$. In addition to the total number of partial waves, the $q$ mesh and largest $q$ value in the integral, Eq. (17), are monitored and checked, as well as the ejected-electron energy mesh and maximum ejectedelectron energy for the integration over the single differential cross section.

The fact that the distorted waves are not orthogonal to the initial bound-state wave functions can cause the integral [Eq. (18)] to become too large for certain cases. The problem occurs only when the angular momentum $l$ of a partial wave is equal to a bound-state wave-function angular momentum $l_{0}$. For $l \neq l_{0}$, orthogonality is provided by the different spherical harmonics. For the $l=l_{0}$ case, it would be desirable to obtain orthogonality by solving the differential equation [Eq. (14)] with Lagrange multipliers. For expediency, a less desirable alternative in the spirit of the Schmidt orthogonalization method was chosen here. For the $\lambda=0$ cases, Eq. (18) was modified by subtracting the overlap integral between the distorted wave and the bound-state wave function.

Finally, theoretical methods, including the one described here, are normally used to calculate $\sigma_{1}$, the cross section for the ejection of one electron from a given subshell. To compare with $\sigma_{-}$, multiple ionization cross sections with weights appropriate to the total number of electrons produced must be included. Multiple ionization primarily occurs through three basic mechanisms occurring either alone or in combination. These mechanisms are (a) inner-shell ionization accompanied by Auger processes, (b) direct ejection of more than one electron, mostly from the same shell, and (c) simultaneous direct ionization by and charge transfer to the projectile. For instance, double ionization of the $\mathrm{Ne} 2 p$ orbital occurs mainly through the ionization of a $1 s$ electron accompanied by an Auger process filling the $1 s$ hole, direct ejection of two $2 p$ electrons, or ejection of a $2 p$ electron and another $2 p$ electron captured by the incident proton. Each of these mechanisms is significant at different proton energies
(DuBois, 1984; DuBois, Toburen, and Manson, 1984).

Table I summarizes theoretical results for $\mathrm{H}, \mathrm{He}, \mathrm{Ne}$, and Ar. As will be shown later, these calculated data agree well at high energies with experimental values for $\mathrm{H}, \mathrm{He}$, and $\mathrm{Ne}$, but not so well for Ar. For distortedwave (first-order perturbation theory) calculations of ionization cross sections for atoms with many bound electrons, accuracies are not better than 10-20\%.

\section{B. Relationship between proton- and electron-impact data}

Consistency checks can be performed on experimental and theoretical cross sections by comparing data for different projectiles. One such comparison can be made for proton- and electron-impact ionization. The projectile dependence enters through the $q_{\min }$ of Eq. (17) and the $(z / v)^{2}$ term of Eq. (16), which is the same for equalvelocity electrons and protons. The value of $q_{\max }$ in Eq. (17) can be set to infinity without significantly affecting integrated cross sections because the integrand of Eq. (17) diminishes as a high power of $q$ at large $q$. The minimum $q$ occurs for $\theta_{p}=0$. Consequently from Eq. (10), one gets

$$
\hbar^{2} q_{\min }^{2}=2 m_{p}\left[E_{p}+E_{p}^{\prime}-2\left(E_{p} E_{p}^{\prime}\right)^{1 / 2}\right] \text {, }
$$

where $E_{p}$ is the initial and $E_{p}^{\prime}$ the final kinetic energy relative to the atom for the projectile of mass $m_{p}$. The final energy of the projectile is

$$
E_{p}^{\prime}=E_{p}-\varepsilon,
$$

where

$$
\varepsilon=I_{j}+E_{e} .
$$

In Eq. (21) $I_{j}$ is the ionization potential for the $j$ th atomic subshell of interest. If Eq. (20) is inserted into Eq. (19), it may be seen that

$$
\hbar^{2} q_{\min }^{2}=\frac{\varepsilon^{2}}{v^{2}}\left[1+\frac{\varepsilon}{2 E_{p}}+\cdots\right] .
$$


Consequently, if the energy of the projectile is large enough so that $\varepsilon / E_{p}$ can be neglected, $q_{\min }$ is the same for equal-velocity electrons and protons. As a result, in the high-energy limit, equal-velocity electrons and protons should have identical cross sections.

At lower energies there are several effects that make the total ionization cross section for proton impact larger than that for equal-velocity electron impact. First, while the cross section for the production of slow electrons by fast electrons is the same as for protons of the same speed, the proton can eject more energetic electrons because of its greater energy. Second, the exchange effect between the incident and bound electrons generally reduces the production of ejected electrons, particularly those with maximum available kinetic energy, i.e., about one-half of the incident electron energy (minus the appropriate ionization potential). Third, protons of moderate energy $(<300 \mathrm{keV})$ have a higher probability for multiple ionization (e.g., ejection of two or more electrons from the same shell) than electrons of the same speed (DuBois, Toburen, and Rudd, 1984). In addition, simultaneous ionization and electron capture by the incident proton becomes significant at low proton energies $(<100 \mathrm{keV})$.

At high projectile speed, however, electron-impact cross sections are expected to approach cross sections for ionization by protons (or other bare, heavy ions if the electron-impact cross section is multiplied by $z^{2}$ ) traveling at the same speed. Consequently, electron-impact cross sections should serve as a lower limit to the protonimpact ionization cross sections.

\section{Comparison with photoionization data}

When the exponential function in the form factor given in Eq. (11) is expanded in a power series, the leading term is the dipole transition matrix element,

$$
\lim _{q \rightarrow 0}\left|F_{f i}\right|^{2}=q^{2}\left|\left\langle\chi_{f}^{(-)}(\mathbf{k}, \mathbf{r})|r \cos \theta| \psi_{i}(\mathbf{r})\right\rangle\right|^{2},
$$

which is, in turn, directly related to the photoionization cross section. Here, the final-state orbital of the active electron is assumed to be orthogonal to its initial-state orbital. Based on this fact, Bethe showed (Bethe, 1930; Inokuti, 1971) that after integrating over $q$, the Born cross section [Eq. (9)] can be expanded in an inverse power series of equivalent incident electron energy $T=m_{e} v^{2} / 2$, where $m_{e}$ is the electron mass:

$$
\sigma_{\text {ion }}=\frac{4 \pi a_{0}^{2}}{T / R}\left[A_{\text {ion }} \ln (T / R)+B_{\text {ion }}+C_{\text {ion }} R / T+\cdots\right],
$$

where $a_{0}$ is the Bohr radius. The leading coefficient. of the expansion, $A_{\text {ion }}$, is given by

$$
A_{\text {ion }}=R \int_{0}^{\infty} \frac{d f}{d \varepsilon} \frac{d \varepsilon}{\varepsilon},
$$

where the continuum dipole oscillator strength $d f / d \varepsilon$ is defined by

$$
d f / d \varepsilon=(\varepsilon / R)\left|\left\langle\chi_{f}^{(-)}(\mathbf{k}, \mathbf{r})|r \cos \theta| \psi_{i}(\mathbf{r})\right\rangle\right|^{2} .
$$

The constant $B_{\text {ion }}$ is characteristic of the target but not of the projectile, and $C_{\text {ion }}$ depends both on target properties and on the type of projectile. Equation (24) is known as the Bethe approximation.

When $\left(T / 4 \pi a_{0}^{2} R\right) \sigma_{\text {ion }}$ is plotted as a function of $\ln (T / R)$, the cross-section data should approach a straight line with a slope $A_{\text {ion }}$ and an intercept $B_{\text {ion }}$. Such a plot, known as a Fano plot (Inokuti, 1971), is a powerful tool for elucidating high-energy behavior of both experimental and theoretical cross sections.

Values of the asymptotic slope $A_{\text {ion }}$ for some atoms and molecules are known (Rieke and Prepejchal, 1972) or can be deduced from available photoionization data (Berkowitz, 1979). To apply the Bethe asymptotic form of Eq. (24) to $\sigma_{-}$, the values of $A_{\text {ion }}$ deduced from photoionization data using Eq. (25) have to be increased by the ratio $\sigma_{-} / \sigma_{\text {count }}$ [i.e., $\kappa_{-}$defined by Eq. (8)] because photoionization data correspond to $\sigma_{\text {count }}$. For instance, a $K$ shell ionization of $\mathrm{Ne}$ by $\mathrm{x}$ rays will be counted as one event, whereas it should be multiplied by two in $\sigma_{-}$because the subsequent decay of the $K$ hole through an Auger process produces another ionized electron from the $L$ shell.

Values of $B_{\text {ion }}^{*}$ are more difficult to determine than those of $A_{\text {ion }}$, and accurate results are known only for simple cases (Kim and Inokuti, 1971; Saxon, 1973; Eggarter, 1975; Kim and Cheng, 1978; Douthat, 1979).

As will be shown later, comparisons with electronimpact as well as photoionization cross sections serve as powerful consistency tests for the high-energy behavior of proton-impact ionization cross sections.

\section{Low-energy behavior}

While theoretical methods to handle ionization at high energies are well developed, present theory tells us very little about electron production at low and intermediate energies. By conservation of momentum and energy it can be shown that, for a collision with an atom of mass $m_{A}$, the threshold for transfer of energy equal to the binding energy $I$ is $E_{\mathrm{th}}=(1+r) I$, where $r=m_{p} / m_{A}$. Since $r$ is less than or equal to unity for proton impact, the threshold is one to two times the ionization potential.

Several theoretical discussions of electron-impact ionization (e.g., Wannier, 1953; Rau, 1984) at threshold are available, but recent experimental data seem to support Wannier's theory, which predicts $\sigma \propto(T-I)^{1.127}$, where $T$ is the incident energy. Klar (1982) extended the Wannier theory to ionization by proton impact and obtained the result that $\sigma \sim\left(E_{p}-I\right)^{D}$ where $D \sim 70$ near threshold. Unfortunately, the range of validity of threshold laws is difficult to determine, but is generally very limited. For instance, theoretical calculations based on transient molecular formation at proton energies of a few hundred $\mathrm{eV}$ (SethuRaman et al., 1973) to a few tens of $\mathrm{keV}$ (Winter 
and Lin, 1984) indicate that the electron-ejection cross section in this energy range rises as $\left(E_{p}-I\right)^{D}$ where $D=2-3$.

Because it is difficult to produce and control proton beams of a few tens of $\mathrm{eV}$, no data are available near threshold for proton-impact ionization. Gilbody and Hasted (1957) measured ionization cross sections at energies as low as $400 \mathrm{eV}$, and Latypov and Shaporenko (1973) obtained data as low as $200 \mathrm{eV}$, still considerably above threshold. Utterback and his co-worker (Utterback and Miller, 1961; Utterback, 1963) avoided the problem of beam deflection at low energies by using beams of neutral nitrogen and oxygen and studied ionization down to center-of-mass energies as low as $2-3 \mathrm{eV}$ above threshold. They generally obtained a power-law dependence with $D$ in the range of 2-4.5. Whether these results are also applicable to protons is not known. In the absence of better information, we shall assume that the low-energy dependence for proton impact can be fitted by a power law. As will be discussed below, the few experimental data that are available generally yield $D$ values between 0.7 and 1.5 .

\section{EXPERIMENTAL METHODS}

Three basic methods are used to make measurements of ionization cross sections by proton impact. These all involve a beam of protons passing through a gas target. In the earliest and most direct method, which was first used in electron-impact work, the charged products of the collision are collected on parallel plates on either side of the collision region. Here this will be called the transversefield method; it also is known as the condenser-plate or the parallel-plate capacitor method. Sometimes a magnetic or time-of-flight spectrometer is added to the transverse-field experiment to distinguish the various charge states or dissociation fragments.

The other two methods were designed for different purposes but yield total ionization cross sections as byproducts of more comprehensive measurements. One of these is the measurement of the angular and energy distribution of ejected electrons, data which provide a stringent test of any theory describing electron ejection. The total cross section may be obtained from these data by integrating over the angles and energies. Unlike the other two methods, this one was developed first for proton impact and only later was applied to electron collisions.

The third is the energy-loss method, in which cross sections are determined by measuring the fraction of the incident beam that has lost a specific amount of energy. It was first developed for electron-impact work, but since electrons are typically scattered through large angles, was not a practical way to measure ionization cross sections. For ions, however, the angular distribution of the scattered particles is so strongly peaked in the forward direction that few are deflected out of the beam. Consequent$1 y$, it is possible to determine cross sections from measurements on the energy-loss spectrum of the beam itself, without having to detect any of the collision products. These three methods will be discussed separately below, with emphasis on the most commonly used method.

\section{A. Transverse-field method}

This method was first applied to proton-impact measurements by Goldman (1932) to measure charge transfer and, unsuccessfully, to measure ionization. Keene (1949) was the first to make a successful ionization measurement.

\section{General arrangement of apparatus}

The basic elements of the apparatus are shown in Fig. 1. The ion beam enters the system through the collimator C followed by a collimator suppressor CS to suppress secondary electrons from $C$. The beam goes through the gas cell GC and into a Faraday cup FC. Secondaries from the latter are prevented from escaping either by a positive bias on FC or by a negative bias applied to the Faraday cup suppressor FCS. In some cases electron suppression is accomplished by using a magnetic field or a transverse electric field within the cup. A negative potential is applied to the ion collecting plate IP and a positive potential to the electron collecting plate EP. The guard plates GP keep the field uniform over the measurement region. A grid $G$ is usually placed between the beam and the ion collecting plate to suppress secondary electrons formed at the plate when the ions strike it. In some experiments a magnetic field, generally parallel to the beam, is used to suppress secondaries from the ion collecting plate, thus making the grid unnecessary. Although some experimentalists also use a grid in front of the electron plate, this is not necessary, since the direction of the field is such that secondary electrons cannot escape from that plate.

If the length of the electron and ion collecting plates parallel to the beam is $l$, the target gas density $n$, and the incident beam current $I_{B}$, then assuming that the target gas density is low enough to ensure single-collision conditions, the cross sections for production of positive and

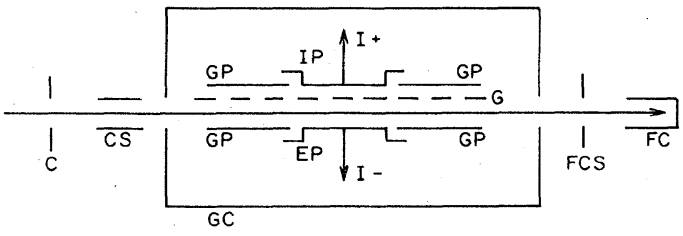

FIG. 1. Schematic diagram of apparatus for the transversefield method of measuring ionization cross sections. $C$ is the beam collimator, CS the collimator suppressor, GC the gas cell, EP, IP, and GP the electron collection plate, the ion collection plate, and the guard plates, respectively, $G$ the secondary electron suppressor grid, FC the Faraday cup, and FCS the Faraday cup suppressor. The currents $I_{+}$and $I_{-}$are measured to obtain the cross sections $\sigma_{+}$and $\sigma_{-}$. 
negative charge are related to the positive ion current $I_{+}$ and electron current $I_{-}$produced by the beam though the relation

$$
\sigma_{ \pm}=\frac{I_{ \pm}}{n l I_{B}}
$$

Although the experiment is simple in concept, in practice many precautions and corrections are necessary if the cross sections are to be determined accurately. These will be discussed in the following sections.

\section{Preparation and collection of the proton beam}

It is a common, almost universal, practice to magnetically analyze the proton beam from the accelerator before allowing it to enter the target cell. This ensures that the beam consists only of protons.

Below about $250 \mathrm{keV}$, electron capture begins to become important enough to cause neutralization of an appreciable fraction of the beam. This may occur along the beam path even before the protons reach the gas cell if a high vacuum is not maintained along the beam line. Neutralization also takes place in the gas cell itself and along the rest of the beam path up to the entrance of the Faraday cup. Two effects result from beam neutralization. First, if a proton in the beam is neutralized any time before it reaches the cup, it is not counted as part of the beam by the current meter or integrator connected to the cup, unless special provision is made to detect neutrals. This causes an error in the measurement of the beam current. Second, if a proton is neutralized before reaching the measuring region, it may still cause ionization but in general will have a different cross section.

The measured ionization cross section can be corrected for these two effects if approximate values are available for $\sigma_{c}$, the capture cross section, and for $\sigma_{+}^{0}$ and $\sigma_{-}^{0}$, which are the cross sections for producing positive ions and electrons, respectively, by neutral atom impact. The modified equations for the cross sections are

$$
\sigma_{ \pm}=\frac{I_{ \pm}}{n l I_{B}} \exp \left(-n l_{2} \sigma_{c}\right)-\sigma_{ \pm}^{0}\left[\exp \left(n l_{1} \sigma_{c}\right)-1\right],
$$

where $l_{1}$ is the effective path length at the target gas pressure between the analyzing magnet and the measuring region, $l_{2}$ is the effective path length from the measuring region to the Faraday cup, and $I_{B}$ is the beam current measured at the Faraday cup. Typically, the corrections for beam neutralization are largest at energies of $1-30$ $\mathrm{keV}$. Few investigators have made this correction, although a $5-\mathrm{keV}$ beam traveling $10 \mathrm{~cm}$ through nitrogen at $3 \times 10^{-4}$ Torr suffers a $15 \%$ neutralization.

To ensure that the Faraday cup collects all of the beam that passes through the target gas, a collimation system is used. After the last collimator aperture, it is important to have a suppressor to prevent the secondary electrons, formed when the beam strikes the aperture edges, from entering the collision region. Such secondaries are mostly of very low energy, and a negative bias on the suppressor (or a positive bias on the aperture) of $50-100 \mathrm{~V}$ is usually sufficient. It is also important to shield the biased suppressor to prevent field penetration into the collision region. For the same reason, the bias on the Faraday cup needs to be shielded, a precaution which some early investigators failed to take.

At low energies, scattering of ions out of the beam becomes a serious problem. Calculations for one geometry (Rudd et al., 1983) indicate that $11 \%$ of a $5-\mathrm{keV}$ proton beam is scattered through a large enough angle to miss the Faraday cup in traversing an argon target at $1 \mathrm{~m}$ Torr.

An important problem results from the necessity of having the beam pass through the transverse field of the collecting plates and guard plates. This field causes a deflection of the beam, which may result in its incomplete collection by the Faraday cup. A compromise must be made between the need for a large enough field to ensure complete collection of all ions and electrons (as discussed in Sec. IV.A.5) and a small enough field to avoid an unacceptable beam deflection.

\section{Determination of target gas density}

For static gas targets, the universal practice is to measure the pressure and temperature and to apply the ideal gas law to determine the density. In studies of ionization cross sections made before about 1965 the McLeod gauge was the standard for pressure measurement. In some cases ionization or Pirani gauges were used; but these were calibrated against a McLeod gauge.

An important disadvantage of the McLeod gauge was not generally known until 1961. To prevent the mercury vapor in the gauge from entering the gas cell, a cold trap is placed between the gas cell and the gauge. Since the trap condenses the mercury vapor, there is a flow of vapor away from the gauge. This causes a pumping effect, resulting in a smaller measured pressure than the actual target gas pressure, which causes the measured cross sections to be too large. The mercury streaming effect had been pointed out by Gaede (1915), but was not generally appreciated until Ishii and Nakayama (1962) showed that it caused an error in pressure readings. The magnitude of the error was shown by Schram et al. (1965) to depend on the diameter of the tubing between the gauge and cold trap, the temperature of the mercury in the gauge, and the molecular mass of the target gas. The error, which is typically only $1-2 \%$ for $\mathrm{H}_{2}$ and $\mathrm{He}$, rises to as much as $40 \%$ for heavier gases. One way to decrease this effect is to cool the mercury reservoir in the gauge, thus reducing the vapor pressure and rate of mercury streaming. Alternatively, the diameter of the tube may be restricted to a capillary size to reduce the flow of mercury vapor. A larger tube normally connecting the gauge to the gas cell is closed off just as the mercury rises to the cutoff point, leaving only the capillary connecting the gauge and gas cell at the moment of measurement.

In another type of gauge, the capacitance manometer, 
which came into general use in the mid to late sixties, a thin, sealed metal membrane separates the target gas from the reference pressure (usually a high vacuum). The position of the membrane varies with the difference in pressure between the two sides. The membrane forms a part of two capacitors which are in a bridge network, the output of which is amplified and read out electronically. While this gauge is not absolute in the sense that the pressure to be measured is directly calculated from measured quantities, the gauge can be calibrated in a dead-weight tester to high accuracy or can be compared with a McLeod gauge. The latter was done by Utterback and Griffith (1966) for the MKS Baratron capacitance manometer, which was found to have good linearity in the $10^{-4}$ to $10^{-3}$ Torr pressure region and an accuracy of $2 \%$ for helium.

Since the capacitance manometer is a differential gauge, either the reference pressure must be negligible compared to the pressure to be measured, or else the reference pressure must be measured and the proper correction made. For best results the capacitance manometer head must be kept at an elevated temperature, usually about $322 \mathrm{~K}$. This causes a difference in gauge pressure and gas cell pressure due to the phenomenon of thermal transpiration. It had been thought that the equation $P_{1} / P_{2}=\left(T_{1} / T_{2}\right)^{1 / 2}$ (Knudsen, 1910) described this effect and could be used to make corrections, but Blaauw et al. (1980) and others showed that while the Knudsen equation holds when an aperture separates the regions at different temperatures, it overcorrects when tubing connects the two regions. For example, they found that while the expected correction was $4.8 \%$ using the Knudsen equation, the actual difference in pressure between regions connected by tubing was only about $2 \%$.

Target gas purity is not usually a problem, as most common gases are available from compressed-gas suppliers at $\mathbf{9 9 . 9 \%}$ purity or better. Precautions must be taken, of course, to use regulator valves, leak valves, and gas lines that have no leaks and that do not introduce vapors of oil, grease, rubber, or other foreign substances.

In most experimental arrangements the gas cell volume is large and the entrance and exit apertures for the beam are small. If this is the case, static gas conditions may be assumed. In some cases, however, a significant flow of gas in the target cell leads to the need for end corrections if the measurement region is near the escape holes for the gas.

\section{Path length}

In the transverse-field method the path length $l$ in the cross-section equation is usually defined by the length, in the beam direction, of the collecting plates. Guard plates at either end of the collecting plates maintain a uniform field and avoid edge effects.

If the electrons or slow ions to be collected have a component of velocity in the forward direction, some of those formed near the forward edge of the collecting plate will follow trajectories past the edge of the plate and will not be collected. In compensation, those formed along the beam path before the collecting plate region do reach the collector, but this compensation is not complete if a significant number of trajectories have components parallel to the beam which are greater than the length of the collecting plates. A change in the effective path length in the gas results. This problem will be examined further in the next section.

An effect that is not generally appreciated can cause ions or electrons to be collected from regions outside the length of beam adjacent to the measuring plates. If a biased suppressor or Faraday cup is used, a longitudinal electric field exists along the beam. If target gas is present in this region, ions or electrons (depending on the polarity of the field) formed in the field are projected along the beam into the measuring region. This source of unwanted current can be eliminated either by evacuating the region near the cup or suppressors or by providing a large field in the collecting region, so that such charges are swept out to a guard plate before reaching the collecting plates.

\section{Electron and ion collection}

The vast majority of collisions with atoms result in the ejection of very slow recoil ions. The energy distribution of these ions is described approximately by a calculation using the screened Coulomb potential (Schiff, 1949). For proton impact on a target atom of mass $m_{A}$ and nuclear charge $Z$, the cross section for giving a recoil energy $E_{r}$ to the target is

$$
\begin{aligned}
\left(d \sigma / d E_{r}\right)_{\text {recoil }}= & 4 \pi a_{0}^{2} R^{2} Z^{2} T^{-1} \\
& \times\left[E_{r}+R\left(1+Z^{2 / 3}\right) m_{e} / m_{A}\right]^{-2} m_{e} / m_{A} .
\end{aligned}
$$

To obtain the cross section for producing an ion of energy $E_{r},\left(d \sigma / d E_{r}\right)_{\text {recoil }}$ must be multiplied by the ionization efficiency. In this derivation the momentum given to the ejected electron has been ignored, so the value of $E_{r}$ represents the upper limit of the recoil energy. The dependence on $E_{r}$ expressed in Eq. (28) has been verified (Crooks, 1974) for energies from a few eV to about 100 eV. This equation predicts that for a helium target, for example, $99 \%$ of the recoil ions have energies less than $0.5 \mathrm{eV}$; for argon targets, $99 \%$ have less than $0.15-\mathrm{eV}$ energy.

There is therefore no difficulty in collecting slow ions from collisions with monatomic targets, but for a molecular target which can dissociate in the collision, the fragments may be ejected with energies up to about $20 \mathrm{eV}$ (see, for example, Edwards et al., 1977). Even in this case, the application of a moderate potential difference between the collecting plates is sufficient to collect all ions.

Electrons present a more difficult problem, since they are ejected in the collision with a greater range of ener- 
gies. While the distribution is peaked at $0 \mathrm{eV}$, a significant fraction of electrons have velocities up to twice the velocity of the projectile and a small fraction even higher. The exponential model (Rudd, 1979) gives a fair approximation to the distribution at the high-energy end of the spectrum. According to this model, the ejection energy above which there is only a fraction $f$ of the ejected electrons is given by

$$
E=(I T)^{1 / 2} \ln (1 / f),
$$

where $T=E_{p} / 1836$ and $I$ is the binding energy of the target atom. This yields the result, for example, that $5 \%$ of electrons ejected in a $5-\mathrm{MeV} \mathrm{H}^{+}+\mathrm{He}$ collision have energies above $775 \mathrm{eV}$.

The angular distribution of electrons ejected in lowenergy collisions is peaked slightly in the forward direction but changes to one peaked strongly in the forward direction for intermediate energies and then becomes peaked near $90^{\circ}$ at high energies (e.g., Rudd et al., 1979).

Continuum electron capture (see, for example, Rudd and Macek, 1972) results in a significant number of electrons being ejected in the forward direction with velocities comparable to that of the projectile velocity. The fraction is estimated to be as much as $30 \%$ for $(100-500)-\mathrm{keV}$ protons (Kim, 1975a). The collecting field should be great enough to cause the trajectories of such electrons to reach the plane of the electron collecting plate before the electron traverses a forward distance equal to the length of the guard plate, as mentioned in Sec. IV.A.4. Since most investigators do not give the dimensions of their electrode systems nor the collecting potentials used, it is difficult to judge whether or not their electron collection was reasonably complete. The usual test is to plot electron current against collection voltage. It is generally assumed that if the current levels off, saturation has been reached. For electron collection, however, the curves rarely attain zero slope, and there is no way of knowing what fraction of the electrons are actually being collected. It is better to utilize known information about the energy distributions of ejected electrons to calculate the electrode biases needed for nearly complete collection. Furthermore, since the energy and angular distributions of electrons depend strongly on proton energy, the biases should be adjusted as the impact energy changes. However, the collecting field must not be made too large since, as noted earlier, a large field may cause an unacceptable deflection of the beam.

When a grid is used for electron suppression at the positive ion plate, it is necessary to make a correction to the measured ion current for the transparency of the grid. The transmission for ions has usually been taken to be the same as the geometrical transmission, but this is only approximately true because of the deflection of ion trajectories by the potentials of the grid and plate. Rudd et al. (1983) showed that the actual transmission $t$ is related to the geometrical transmission $t_{0}$ by the relation

$$
t=\left[1+\left(t_{0}^{-1}-1\right)\left(V_{g} / b V_{i}\right)^{n}\right]^{-1},
$$

where $V_{i}$ and $V_{g}$ are the potentials of the ion plate and grid, respectively, relative to the beam, and $b$ is the ratio of the beam-to-plate distance to the beam-to-grid distance. This equation is valid when the initial ion energy is small compared to its energy at the grid or plate. When the exponent $n$ was taken to be equal to $\frac{1}{2}$, a good fit to experimental data was found. Typically, the value of the opacity, $1-t$, is $50-80 \%$ greater than the geometrical blocking, $1-t_{0}$. Then if $t_{0}=90 \%$, the use of $t_{0}$ in place of $t$ would cause an error of 5-8\%.

\section{Spurious currents}

In the measurement of ion currents and, to an even greater extent, electron currents, care must be taken to avoid spurious currents. Some of the possible sources of these currents are discussed in the following paragraphs.

\section{a. Secondary electrons from slow ions striking the grid}

Even though most of the ions pass through the grid, those that strike it produce secondaries, which must be taken into account in determining the current of electrons from the collision. At high energies this is a small correction, at most $1-2 \%$, but at low energies electron capture by protons causes many more positive ions to be produced than electrons. In fact, $\sigma_{+}$may be an order of magnitude greater than $\sigma_{-}$, so even though the secondary current from the grid may be only $1 \%$ of the ion current, it may be $10 \%$ or more of the electron current.

Since the grid is at a potential energy maximum for negative charges, electrons produced there may fall to either plate. Because most of the ions strike the grid on the side away from the ion collecting plate, it seems likely that most of the secondaries would go to the electron collecting plate. To make a correction for this spurious current, the value of the secondary emission coefficient must be known. Ghosh and Sheridan (1957) found that this coefficient varies with the energy and mass of the impinging ion and also depends on the composition and cleanliness of the surface. Thus it is best to determine the secondary emission coefficient for the specific surfaces used. Rudd et al. (1983) described one method for doing this in situ.

\section{b. Photoelectrons}

Ultraviolet photons in the wavelength range of $120-30$ $\mathrm{nm}$ [i.e., (10-40)-eV energies] produce photoelectrons on most metal surfaces. If the proton beam causes excitation in the target gas or on solid surfaces leading to the emission of photons in that wavelength region, photoelectrons will be generated on all surfaces on which the light falls. As shown by Rudd et al. (1983) and Rudd (1984), this does not usually produce an appreciable error because the cross sections for producing uv photons are too low. 


\section{c. Secondary electrons from surfaces struck by scattered beam particles}

Protons in the beam may be scattered from the edges of collimator apertures and also from collisions with the target gas. If these scattered protons strike metal surfaces such as the grid or the beam suppressor, secondary electrons are produced. It is difficult to estimate the scattering from the collimator, but it can be minimized. This is accomplished by making the edges of the aperture as sharp as possible, thus limiting the area from which scattering into the measuring region can take place, and by having a shield past the collimator just large enough to let the main beam pass but small enough to stop most scattered particles. The scattering from target gas atoms (which can be estimated, for example, by calculating the scattering from a screened Coulomb potential) is appreciable only for very low proton energies and for heavy targets, and even then, except for unusual geometries, should not produce many secondary electrons.

\section{d. Sputtering and reflection of ions from the Faraday cup}

If an appreciable fraction of the protons striking the bottom of the Faraday cup are reflected, either as ions or as neutrals, or if they sputter energetic ions or atoms from the surface, additional ionization may be caused as these particles pass back through the target gas. The sputtering yield for protons is less than $1 \%$ for most materials (Thomas, 1985) and therefore should not be an important problem. However, the reflection coefficient for protons rises to a value greater than $10 \%$ below 5 or $10 \mathrm{keV}$ (Thomas, 1985) and therefore could be a serious problem. Another possible source of spurious ionization is from the radiation emitted from the Faraday cup on bombardment by the ion beam. It is well known that radiation in the uv and $\mathrm{x}$-ray regions results from ion impact on solids, but little or no data exist that would enable us to estimate the magnitude of the effect and of its influence on an experiment.

\section{Variations of the transverse-field method}

Because it is difficult to control spurious electrons, Gilbody and Lee (1963) avoided making direct measurements of electron currents by measuring the ion current to one plate and the total current to both plates. The latter current is a measure of the electron capture. Then the cross section $\sigma_{-}$is determined from $\sigma_{+}$and $\sigma_{c}$, using Eq. (5). In other investigations (e.g., Gilbody and Lee, 1963) only $\sigma_{+}$was measured, and values of $\sigma_{c}$ from other authors were used to obtain $\sigma_{-}$. Since this approach involves taking differences between two quantities, each of which has its own uncertainty, the possibilities for error are increased, especially at low energies where $\sigma_{-}$is much smaller than the other two cross sections.

When the desired target is not stable or not easily available as a static gas, experimenters have used the crossed- beam method. Fite et al. (1960) and Gilbody and Ireland (1963) were among the early users of this technique for proton ionization measurements. The details of this technique are discussed elsewhere (e.g., Bederson, 1968). The path length of the beam in the active region is defined by the target beam size rather than by the length of a plate, so end effects and the production of secondary products near biased suppressors is usually not a problem. However, since the beams may not be uniform, the overlap integral of the densities of the beams must be evaluated by determining the profile of both beams. An error is introduced if the beams shift in position or change their profiles between the times the overlap integral is measured and the cross sections are measured. Since the target densities are usually very small, beam neutralization problems are avoided. Unfortunately, a way to normalize the cross sections must be devised, since the absolute target density in the beam is difficult to measure.

To obtain more detailed information about the products of ionization, a provision to measure the mass-to-charge ratio $(m / q)$ of the residual ions is often incorporated in the transverse-field apparatus. In fact, in the first successful proton ionization measurement, Keene (1949) did an auxiliary experiment of this kind to determine the fraction of $\mathrm{H}_{2}$ that was dissociated in ionizing collisions. Others, such as Wexler (1964), used $m / q$ analysis to determine the fraction of ions in various charge states. Usually only relative values of cross sections for production of various charge states are measured by this method, and absolute values are obtained by comparison to known values of $\sigma_{+}$using Eq. (6).

Afrosimov et al. (1969) pioneered the coincidence technique for measuring specific ionization processes. By analyzing the charge states of the projectile and of the secondary products from the same collision, they were able to measure separately the cross sections for ionization, ionization with dissociation, double ionization, capture, capture with dissociation, capture with ionization, and double capture in $\mathrm{H}^{+}+\mathrm{H}_{2}$ collisions. The total ionization cross sections of interest here may be determined by taking the proper combination of these specific cross sections.

At low impact energies (say, below $3 \mathrm{keV}$ ) it becomes very difficult to find a transverse field that is sufficiently strong to collect all ions and electrons, but not so strong that it deflects part of the primary beam outside the detection region. Latypov and Shaporenko (1972) developed a system in which the ion beam and the collecting field are pulsed so that they are not both on at the same time. The beam passes through the target in a field-free region and produces ions and electrons. Then the beam is turned off and the collecting field is pulsed on long enough to propel the ions into an $m / q$ analyzer.

\section{B. Integration of differential cross sections}

In the early sixties a method was developed for studying the angular and energy distribution of electrons eject- 
ed in ion-atom collisions (Kuyatt and Jorgensen, 1963; Rudd and Jorgensen, 1963). Double differential cross sections (DDCS) were measured over wide ranges of angle and energy' to allow integration over both variables to give total electron-ejection cross sections. These DDCS provide much detailed information about ionization and have been useful in guiding theoretical advances in our understanding of this process. Here our only interest in this type of measurement is in its capacity to yield total electron-ejection cross sections as a by-product of a much more comprehensive measurement.

Figure 2 shows the basic components of the apparatus for this type of measurement. The ion beam from an accelerator is collimated by $\mathrm{C} 1$ and C2. Suppressor CS prevents electrons ejected from the collimator edges from entering the target gas. A shield SH stops most of the ions scattered from aperture edges and also prevents the field from the suppressor from penetrating into the target gas. The beam is caught by a Faraday cup FC. Electrons ejected at an angle $\theta$ at the collision center $\mathrm{CC}$ are collimated by $\mathrm{C} 3$ and $\mathrm{C} 4$ before entering the electrostatic analyzer EA, here shown as a parallel-plate analyzer. Other types of analyzers have also been successfully used. For example, Toburen (1974) used a cylindrical mirror analyzer, and Kuyatt and Jorgensen (1963) employed a $127^{\circ}$ electrostatic analyzer. Electrons that pass through the analyzer are accelerated to the first dynode of the electron multiplier detector ED. The energy spectrum of ejected electrons is obtained by running through the proper range of analyzer voltages. After measurements have been made at a sufficient number of angles, numerical integration of the DDCS yields the total ionization cross sections.

The major source of error in this method for obtaining total cross sections lies in the fact that most of the contribution to the integral over ejected-electron energy comes from low electron energies, typically 0 to $20 \mathrm{eV}$. At energies below $10 \mathrm{eV}$ considerable variability has been observed by different investigators and even by the same investigator at different times. In some cases the measured cross section is too large due to the generation of spurious electrons from ions striking surfaces. More often the cross section is too small because very-low-energy electrons sometimes fail to travel through the analyzer and reach the detector. Even very small magnetic or electric fields are sufficient to deflect the trajectories to an unacceptable extent. Magnetic shielding against the Earth's magnetic field or cancellation of the field by Helmholtz coils is required. Stray electric fields may arise from electrons residing on insulating surfaces, such as oil or oxide films on target cell walls or on collimator slits. In some experiments the electrons are pre-accelerated just before entering the electrostatic analyzer to make them less susceptible to stray fields on their passage through the analyzer. Care must be taken to ensure that the field from the acceleration slit does not leak out into the supposedly field-free region between collimators C3 and C4, since this would cause a distortion of the effective collection geometry. Pre-acceleration can also deflect trajec-

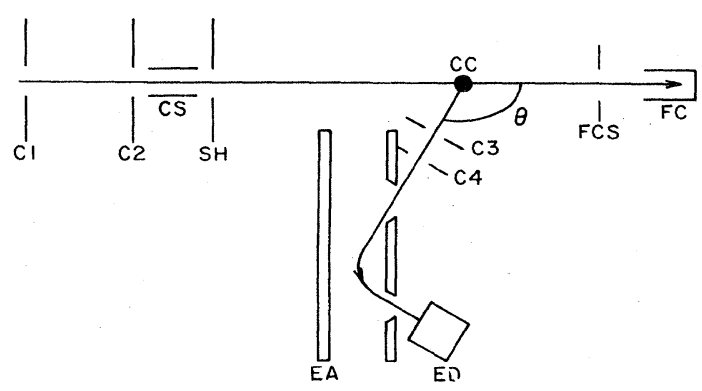

FIG. 2. Schematic diagram of the apparatus for making differential cross-section measurements of electron ejection. C1 and $\mathrm{C} 2$ are the beam collimators, CS the collimator suppressor, SH the shield, CC the collision center, FC the Faraday cup, FCS the Faraday cup suppressor, C3 and C4 the collimators for the ejected electrons, EA the energy analyzer, and ED the electron detector. The angle $\theta$ is variable.

tories of electrons, causing them to be lost.

It is difficult to calculate the error in the total cross section caused by the loss (or gain) of low-energy electrons, but a simple approximation using the Thomson equation for cross sections (Thomson, 1912) yields for the fraction $f$ of electrons with energies below an energy $E$, $f=E /(E+I)$, where $I$ is the binding energy. Thus, if half of the electrons below, say, $4 \mathrm{eV}$ were lost in $\mathrm{H}^{+}+\mathrm{H}_{2}$ collisions, the integrated cross section would be too small by $10 \%$.

A different approach to the problem of analyzing and collecting low-energy electrons was devised by Toburen and Wilson (1975), who replaced the electrostatic analyzer by a time-of-flight (TOF) analyzer. This system has the advantage that it is a more open structure, with less chance for insulating surfaces to cause problems. Furthermore, the electrons do not have as long a path to negotiate. While the resolution of the TOF analyzer is excellent for slow electrons, the resolution decreases as the energy is increased. For the analyzer used by Toburen and Wilson, the highest usable energy was about $100 \mathrm{eV}$. As it is difficult to measure absolute values of cross sections using this type of analyzer, TOF measurements are normalized to measurements made at an intermediate electron energy with an electrostatic deflection analyzer. The combination of normalized TOF measurements at low energies and electrostatic analyzer measurements at higher energies yields electron spectra of high accuracy.

Except for distortion created by stray fields (already mentioned), the path length in the gas is well defined by the slits C3 and C4. The gas pressure measurement falls heir to the same problems previously mentioned, and a correction for neutralization of the beam must be made at low energies just as with the transverse-field method. An additional correction is required for the absorption of electrons by the gas between the collision center and the detector due to the long path. In the work of Stolterfoht (1971a,1971b), the absorption and neutralization corrections are reduced or eliminated by using a directed gas beam target. However, these measurements must be nor- 
malized against static-gas measurements at each angle, since the product of gas density and path length is not easily measured for the gas beam and varies with angle.

The efficiency of electron multipliers for the detection of single electrons is generally $70-100 \%$ and must be measured for accurate work. It is beyond the scope of this review to discuss the several ways this has been done, but it should be noted that the measurement of this quantity typically introduces an additional uncertainty of about $10 \%$ into the cross-section measurement.

Integration over energy and angle of the ejected electrons is performed numerically and usually poses no special problems except when structure appears in the distribution. Peaks in the energy distribution resulting from autoionization and the Auger effect (see, for example, Rudd and Macek, 1972) generally do not add much area to the energy integral. At $\mathrm{MeV}$ impact energies, however, the binary encounter peak becomes very sharp, making it more difficult to determine the integral accurately. Likewise, failure to integrate accurately over the forward peak caused by electron capture to the continuum may introduce an additional error. This problem is not as serious as it might seem because the integration over angle involves multiplication by $\sin \theta$. Nevertheless, it is important to measure to as small an angle as possible to minimize this error.

\section{Energy-loss method}

Instead of studying the secondary products of the collision, in the energy-loss method the beam itself is energy analyzed after passing through the target gas. The energy-loss spectrum can then be used to obtain cross sections for any process that causes a specific loss of energy of the beam particles. While energy-loss spectroscopy had been well developed in electron-impact work and had been used for ion collisions, it was not used to measure proton-impact ionization cross sections until 1969 (Park and Schowengerdt, 1969). Although the method is best suited for processes such as excitation to metastable states, this group also measured ionization cross sections by integrating over the energy region corresponding to energy losses above the ionization threshold. They solved the formidable problems associated with analyzing beams of energies up to $200 \mathrm{keV}$ to a resolution of $2 \mathrm{eV}$ or better by decelerating the beam before energy analyzing it. Deconvolution techniques were also used to improve the effective resolution.

The apparatus used by Park and his collaborators is shown in Fig. 3. After acceleration, ions from the ion source pass through the target. A magnetic analyzer then removes neutrals or other undesired charge states from the beam. Deceleration to an energy selected by $\Delta V$ (usually $2 \mathrm{keV}$ ) takes place before the beam enters the $127^{\circ}$ electrostatic analyzer. The analyzer potential is held constant while a sweep voltage is applied to the deceleration system, giving the desired energy-loss spectrum. Since the deceleration system is referenced to the accelerator

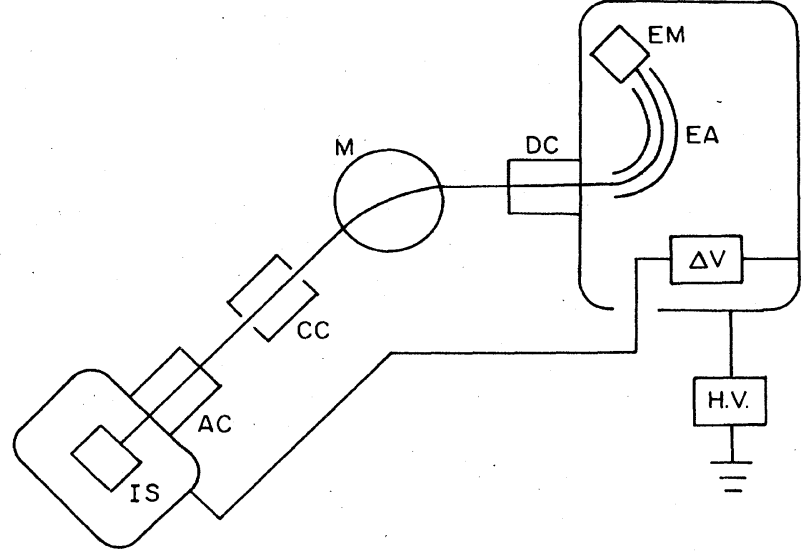

FIG. 3. Schematic diagram of the apparatus for making energy-loss measurements. IS is the ion source, AC the acceleration column, $\mathrm{CC}$ the collision chamber containing the target gas, $\mathbf{M}$ the deflecting magnet, $\mathbf{D C}$ the decelerating column, EA the energy analyzer, EM the electron multipler detector, $H V$ the high voltage used for accelerating the beam, and $\Delta V$ an offset voltage.

terminal, any ripple or fluctuation in the acceleration voltage has little or no effect on the results. Sweeps are made of the energy-loss spectrum with and without the target gas present. By combining these measurements with the measured target gas density and length, differential cross sections are obtained for energy losses to any energy within the ionization continuum. Integration over the entire continuum yields total ionization cross sections.

This method can be used to measure total cross sections because the vast majority of ion beam particles are deflected through only very small angles during atomic collisions. Nevertheless, a possible source of error is the failure to collect all of the scattered beam within the angular acceptance of the analyzer. The angular deflection of the beam particles that have made collisions close enough to cause ionization may be somewhat greater than that of particles that have made elastic collisions. Therefore the fact that only a small fraction of the total beam is lost is insufficient to ensure the complete collection of the part of the beam that made ionizing collisions. Put another way, the energy-loss spectrum of the collected protons must be the same as the spectrum of all the protons traversing the target gas. In recent work this group has developed the capability of measuring the angular dependence of their cross sections. When these are integrated over angle, this error is avoided (see, for example, Park, 1983).

The energy-loss method is immune to the effects of spurious current such as those caused by secondary electrons from surfaces, since these do not affect the energy loss of beam particles. Likewise, neutralization of the beam has no effect on the results. Since the energy loss is a small fraction of the beam energy, protons that have made ionizing collisions have the same probability of being neutralized as those that have not. Thus the ratio from which the cross section is calculated is not affected. 
These are important advantages, but it must be kept in mind that the cross sections obtained using the energyloss method do not describe exactly the same set of specific processes as the other two methods, e.g., they do not include any of the processes that involve a change in the charge state of the beam particle. Also, knowledge of an energy loss involving an inner-shell vacancy does not provide information on the number of electrons ejected because different decay schemes are possible. These are often serious sources of discrepancy between measurements of this kind and those made by the other methods. Such discrepancies occur mostly at energies below about $100 \mathrm{keV}$, where electron capture is most likely to occur.

\section{SURVEY AND CRITIQUE OF EXISTING EXPERIMENTAL DATA}

In preparing this review, the literature was surveyed to obtain a complete list of all total ionization cross-section data published for proton impact on gases. Completeness, of course, is an ideal which is approached but probably not realized. Of great assistance in making the survey were the following bibliographic lists.

(1) “Atomic Data for Controlled Fusion Research" (C. F. Barnett et al., 1977).

(2) Oak Ridge National Laboratory Report No. ORNL-5921, a listing for the period 1978-1981.

(3) Lockheed DIALOG, computer listing for the period 1967-1982.

(4) CIAMDA 80, International Atomic Energy Agency, Vienna, 1980.

(5) NBS Special Report 593, covering the period 1970-1979.

Copies of all of the known papers containing crosssection data were gathered. Tabular data, where available, were entered directly into a computer file. If the data were only presented graphically, they were read from the published graph using standard digitizing techniques. The error introduced by this transcription is estimated at 3-5\%. In some cases partial cross sections for specific processes were combined to obtain the total ionization cross sections. In one case the data were presented in the form of relative cross sections, but enough additional information was given to allow calculation of the absolute cross sections. The data sets were sorted according to target species and plotted. Table II lists all of the data sets by target, along with the energy range, the basic method used, and comments on the experiments.

For each data set, descriptions of experimental apparatus and technique were studied to identify possible sources of systematic error. These errors are noted along with other comments in Table II. A number of data sets listed in this table were excluded from the figures and from the averaging process, either because they were normalized to earlier data, and thus did not represent independent data, or because they had already been reported elsewhere. Data excluded were those of Afrosimov et al. (1967), Wexler (1964), the argon data of Afrosimov et al. (1958b), the CO data of Poulizac et al. (1966), the $\mathrm{N}_{2}$ and $\mathrm{O}_{2}$ data of Desesquelles et al. (1966), and the krypton data of Levchenko et al. (1973).

As noted earlier, the measurements taken by the energy-loss method do not include any processes leading to a change of charge state of the incident ion, and therefore the electron-ejection cross sections are underestimated, especially at low energies where simultaneous electron capture and ionization are likely. The method can also yield too low a cross section if beam ions are scattered outside the angular acceptance of the analyzer. Therefore, in the fitting process, the data of Park et al. $(1969,1971,1977)$ were omitted for atomic hydrogen below $35 \mathrm{keV}$, for helium below $60 \mathrm{keV}$, and for oxygen below $70 \mathrm{keV}$.

In a few cases adjustments were made to the published data. Collins and Kebarle (1967) suggested that their cross sections should be reduced by the factor 1.4 due to end effects (although this correction was not made in the data reported), and their suggestion was followed here. Gilbody and Lee (1963) were unable to calculate $\sigma_{-}$data for krypton from their $\sigma_{+}$cross sections since electron capture data were not then available. Here the capture cross sections of Williams and Dunbar (1966) were used to make this calculation.

Hooper (1961) stated that the positive and negative collected currents in their experiment were the same in all cases. This seems to indicate an error below $250 \mathrm{keV}$, since electron capture must have produced an excess of positive current by as much as $12 \%$. This error also shows up when their cross sections are compared to other measurements. In nearly every case their values are too high, relative to other measurements, by an amount that increases as the energy decreases below $250 \mathrm{keV}$. Therefore their data are assumed to be for $\sigma_{+}$, and the cross sections for electron capture given in the compilation of Barnett et al. (1977) have been subtracted for energies of $250 \mathrm{keV}$ and lower. Even with this correction, Hooper's data appear to be somewhat higher than those of other investigators at $150-250 \mathrm{keV}$. This is probably due to deflection of the beam by the rather large transverse field that was used. A calculation of the beam deflection expected from the dimensions of their apparatus and the collection voltage used indicates a deflection greater than the radius of their Faraday cup. Hooper (1961) noted in his thesis that it was sometimes necessary to remove the collection voltage while measuring the beam current. Other investigators (e.g., McNeal, 1970) have noted similar problems at their lowest energies.

\section{PROCEDURE FOR CHOOSING RECOMMENDED VALUES}

\section{A. Systematic errors}

For several of the targets, especially at low energies, the discrepancies among the various data sets exceed the combined uncertainties quoted by the authors. This clearly 
TABLE II. Total proton ionization cross-section data available.

\begin{tabular}{|c|c|c|c|}
\hline $\begin{array}{c}\text { Energy } \\
(\mathrm{keV})\end{array}$ & Method $^{\mathrm{a}}$ & Investigator & Comments \\
\hline 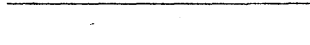 & \multicolumn{2}{|c|}{ Monatomic targets } & \\
\hline \multicolumn{4}{|l|}{ Atomic Hydrogen $(\mathrm{H})$} \\
\hline $7-40$ & $T, G$ & Fite et al., 1960 & g \\
\hline $60-370$ & $T, G$ & Gilbody and Ireland, 1963 & $\mathrm{e}, \mathrm{i}, \mathrm{v}$ \\
\hline $25-200$ & $E$ & Park et al., 1977 & $\mathrm{jj}, \mathrm{kk}$ \\
\hline $38-1500$ & $T, G, C$ & Shah and Gilbody, 1981 & $\mathrm{~m}$ \\
\hline $20-200$ & $E$ & Park, 1983 & \\
\hline \multicolumn{4}{|l|}{ Helium (He-3) } \\
\hline $3-30$ & $T, S$ & Becker and Scharmann, 1969 & $f, o, x$ \\
\hline \multicolumn{4}{|l|}{ Helium ( $\mathrm{He}-4)$} \\
\hline $5-35$ & $T, S, M$ & Keene, 1949 & $\mathrm{c}, \mathrm{h}, \mathrm{r}, \mathrm{dd}$ \\
\hline $0.4-40$ & $T, S$ & Gilbody and Hasted, 1957 & $t, v, 11$ \\
\hline $20-180$ & $T, S$ & Fedorenko et al., 1960 & $\mathrm{c}, \mathrm{j}, \mathrm{r}, \mathrm{t}, \mathrm{v}$ \\
\hline $150-1000$ & $T, S$ & Hooper, 1961 & $\mathrm{~d}, \mathrm{e}, \mathrm{g}, \mathrm{s}, \mathrm{t}, \mathrm{v}$ \\
\hline $10-175$ & $T, S$ & Solov'ev et al., 1962 & $\mathrm{c}, \mathrm{j}, \mathrm{r}, \mathrm{t}, \mathrm{v}$ \\
\hline $120-440$ & $T, S$ & Gilbody and Lee, 1963 & $\mathrm{c}, \mathrm{k}, \mathrm{w}$ \\
\hline $50-150$ & $I$ & Rudd and Jorgensen, 1963 & h \\
\hline $1000-3750$ & $T, S, M$ & Wexler, 1964 & aa \\
\hline $10-140$ & $T, S$ & De Heer et al., 1966 & c \\
\hline $20-120$ & $T, S$ & Desesquelles et al., 1966 & $c, e, g, p, t, v$ \\
\hline $100-300$ & $I$ & Rudd et al., 1966 & h,cc,hh \\
\hline $1000-3000$ & $T, S$ & Pivovar and Levchenko, 1967 & ee \\
\hline $3-30$ & $T, S$ & Becker and Scharmann, 1969 & $f, o, x$ \\
\hline $25-125$ & $E$ & Park and Schowengerdt, 1969 & $\mathrm{jj}$ \\
\hline $150-1000$ & $T, S, M$ & Puckett and Martin, 1970 & $\mathrm{e}, 1, \mathrm{z}$ \\
\hline $300-500$ & $I, G$ & Stolterfoht, 1971a & cc,ii \\
\hline $0.3-1$ & $T, S, M$ & Latypov and Shaporenko, 1973 & $\mathrm{c}, \mathrm{e}, \mathrm{mm}, \mathrm{nn}, \mathrm{oo}$ \\
\hline $300-1500$ & $I$ & Toburen, 1975 & $\mathrm{pp}$ \\
\hline $4200-5000$ & $I, G$ & Stolterfoht, 1975 & \\
\hline $5-100$ & $I$ & Rudd and Madison, 1976 & ii \\
\hline $1440-5120$ & $T, S$ & Hvelplund et al., 1980 & $e, n, f f$ \\
\hline $5-4000$ & $T, S$ & Rudd et al., 1983 & u \\
\hline \multicolumn{4}{|l|}{$\mathrm{Neon}(\mathrm{Ne})$} \\
\hline $0.9-40$ & $T, S$ & Gilbody and Hasted, 1957 & $\mathrm{t}, \mathrm{v}, 11$ \\
\hline $5-200$ & $T, S$ & Fedorenko et al., 1960 & $\mathrm{c}, \mathrm{j}, \mathrm{r}, \mathrm{t}, \mathrm{v}$ \\
\hline $150-1100$ & $T, S$ & Hooper, 1961 & d,e,g,s,t,v \\
\hline $15-180$ & $T, S$ & Solov'ev et al., 1962 & $c, j, r, t, v$ \\
\hline $105-420$ & $T, S$ & Gilbody and Lee, 1963 & $\mathrm{c}, \mathrm{k}, \mathrm{w}$ \\
\hline $800-3750$ & $T, S, M$ & Wexler, 1964 & aa \\
\hline $10-140$ & $T, S$ & De Heer et al., 1966 & $\mathrm{c}$ \\
\hline $30-60$ & $T, S$ & Desesquelles et al., 1966 & $c, e, g, p, t, v$ \\
\hline $1000-3000$ & $T, S$ & Pivovar and Levchenko, 1967 & ee \\
\hline $40-100$ & $T, S, M$ & Collins and Kebarle, 1967 & $b, c, g, p, y$ \\
\hline $50-300$ & $I$ & Crooks and Rudd, 1971 & \\
\hline $0.2-1$ & $T, S$ & Latypov and Shaporenko, 1973 & $\mathrm{c}, \mathrm{e}, \mathrm{mm}, \mathrm{nn}, \mathrm{oo}$ \\
\hline $300-1500$ & $I$ & Toburen et al., 1978 & pp \\
\hline $5-4000$ & $T, S$ & Rudd et al., 1983 & $\mathrm{u}$ \\
\hline \multicolumn{4}{|l|}{ Sodium $(\mathrm{Na})$} \\
\hline $20-100$ & $T, G$ & O'Hare et al., 1975 & $e, f, o, t, v, x$ \\
\hline \multicolumn{4}{|l|}{ Argon (Ar) } \\
\hline $0.4-40$ & $T, S$ & Gilbody and Hasted, 1957 & $t, v, 11$ \\
\hline $5-180$ & $T, S$ & Afrosimov et al., $1958 \mathrm{~b}$ & $\mathrm{c}, \mathrm{j}, \mathrm{r}, \mathrm{t}, \mathrm{v}$ \\
\hline $5-180$ & $T, S$ & Fedorenko et al., 1960 & $\mathrm{c}, \mathrm{j}, \mathrm{r}, \mathrm{t}, \mathrm{v}$ \\
\hline $150-1100$ & $T, S$ & Hooper, 1961 & $\mathrm{~d}, \mathrm{e}, \mathrm{g}, \mathrm{s}, \mathrm{t}, \mathrm{v}$ \\
\hline $15-190$ & $T, S$ & Solov'ev et al., 1962 & $\mathrm{c}, \mathrm{j}, \mathrm{r}, \mathrm{t}, \mathrm{v}$ \\
\hline $125-440$ & $T, S$ & Gilbody and Lee, 1963 & $\mathrm{c}, \mathrm{k}, \mathrm{w}$ \\
\hline $1-40$ & $T, S$ & Gordeev and Panov, 1964 & $\mathrm{c}, \mathrm{j}, \mathrm{r}, \mathrm{t}, \mathrm{v}$ \\
\hline $1000-3750$ & $T, S, M$ & Wexler, 1964 & aa \\
\hline $10-140$ & $T, S$ & De Heer et al., 1966 & $\mathrm{c}$ \\
\hline
\end{tabular}


TABLE II. (Continued).

\begin{tabular}{|c|c|c|c|}
\hline $\begin{array}{c}\text { Energy } \\
(\mathrm{keV})\end{array}$ & Method $^{\mathrm{a}}$ & Investigator & Comments \\
\hline \multicolumn{4}{|c|}{ Monatomic targets } \\
\hline $25-100$ & $T, S$ & Desesquelles et al., 1966 & $c, e, g, p, t, v$ \\
\hline $5-50$ & $T, C, S$ & Afrosimov et al., 1967 & 1 \\
\hline $1000-3000$ & $T, S$ & Pivovar and Levchenko, 1967 & ee \\
\hline $50-300$ & $I$ & Crooks and Rudd, 1971 & \\
\hline $300-5000$ & $I$ & Gabler, 1974 & \\
\hline $5-50$ & $I$ & Criswell et al., 1977 & c,pp \\
\hline $5-70$ & $I$ & Rudd, 1977 & \\
\hline $250-1500$ & $I$ & Toburen et al., 1978 & pp \\
\hline $5-4000$ & $T, S$ & Rudd et al., 1983 & $\mathbf{u}$ \\
\hline \multicolumn{4}{|l|}{ Potassium (K) } \\
\hline $20-100$ & $T, G$ & McCullough and Gilbody, 1971 & $e, t, v$ \\
\hline $20-100$ & $T, G$ & O'Hare et al., 1975 & $e, f, o, t, v, x$ \\
\hline \multicolumn{4}{|l|}{ Krypton $(\mathbf{K r})$} \\
\hline $8-190$ & $T, S$ & Fedorenko et al., 1960 & $\mathrm{c}, \mathrm{j}, \mathrm{r}, \mathrm{t}, \mathrm{v}$ \\
\hline $10-210$ & $T, S$ & Solov'ev et al., 1962 & $\mathrm{c}, \mathrm{j}, \mathrm{r}, \mathrm{t}, \mathrm{v}$ \\
\hline $95-440$ & $T, S$ & Gilbody and Lee, 1963 & $\mathrm{c}, \mathrm{k}, \mathrm{w}$ \\
\hline $1200-3750$ & $T, S, M$ & Wexler, 1964 & aa \\
\hline $10-140$ & $T, S$ & De Heer et al., 1966 & c \\
\hline $1000-3000$ & $T, S$ & Pivovar and Levchenko, 1967 & ee \\
\hline $40-100$ & $T, S, M$ & Collins and Kebarle, 1967 & $\mathrm{~b}, \mathrm{c}, \mathrm{g}, \mathrm{p}, \mathrm{y}$ \\
\hline $200-1800$ & $T, S, M$ & Levchenko et al., 1973 & 1 \\
\hline $5-4000$ & $T, S$ & Rudd et al., 1983 & $\mathbf{u}$ \\
\hline \multicolumn{4}{|l|}{ Xenon $(\mathrm{Xe})$} \\
\hline $8-170$ & $T, S$ & Fedorenko et al., 1960 & $\mathrm{c}, \mathrm{j}, \mathrm{r}, \mathrm{t}, \mathrm{v}$ \\
\hline $300-2000$ & $I$ & Toburen, 1974 & bb,pp \\
\hline \multicolumn{4}{|c|}{ Diatomic targets } \\
\hline $2-35$ & $T, S, M$ & Keene, 1949 & $\mathrm{c}, \mathrm{h}, \mathrm{r}, \mathrm{dd}$ \\
\hline $12.3-36.7$ & $T, S$ & Fogel' et al., 1955 & g \\
\hline $0.4-40$ & $T, S$ & Gilbody and Hasted, 1957 & $\mathrm{t}, \mathrm{v}, 11$ \\
\hline $5-165$ & $T, S$ & Afrosimov et al., 1958a & $\mathrm{c}, \mathrm{j}, \mathrm{r}, \mathrm{t}, \mathrm{v}$ \\
\hline $9-60$ & $T, S$ & Schwirzke, 1960 & $\mathrm{c}, \mathrm{h}, \mathrm{t}, \mathrm{v}$ \\
\hline $150-1100$ & $T, S$ & Hooper et al., 1961 & d,e,g,s,t,v \\
\hline $10-175$ & $T, S$ & Solov'ev et al., 1962 & $c, j, r, t, v$ \\
\hline $50-100$ & $I$ & Kuyatt and Jorgensen, 1963 & c,h,bb,gg \\
\hline 100 & $I$ & Rudd and Jorgensen, 1963 & h \\
\hline $85-450$ & $T, S$ & Gilbody and Lee, 1963 & $\mathbf{c}, \mathbf{k}, \mathbf{w}$ \\
\hline $1-40$ & $T, S$ & Gordeev and Panov, 1964 & $\mathrm{c}, \mathrm{j}, \mathrm{r}, \mathrm{t}, \mathrm{v}$ \\
\hline $1.5-30$ & $T, S$ & Hollricher, 1965 & $\mathrm{p}, \mathrm{y}$ \\
\hline $10-140$ & $T, S$ & De Heer et al., 1966 & c \\
\hline $100-300$ & $I$ & Rudd et al., 1966 & $\mathrm{~h}, \mathrm{cc}, \mathrm{hh}$ \\
\hline $30-90$ & $T, S$ & Desesquelles et al., 1966 & $c, e, g, p, t, v$ \\
\hline $1000-3000$ & $T, S$ & Pivovar and Levchenko, 1967 & ee \\
\hline $5-50$ & $T, C, S$ & Afrosimov et al., 1969 & $\mathrm{c}, \mathrm{e}, \mathrm{k}, \mathrm{o}, \mathrm{p}$ \\
\hline $300-1500$ & $I$ & Toburen and Wilson, 1972 & bb,pp \\
\hline $5-100$ & $I$ & Rudd, 1979 & \\
\hline $38-1500$ & $T, G, C$ & Shah and Gilbody, 1982 & qq \\
\hline $5-4000$ & $T, S$ & Rudd et al., 1983 & u \\
\hline \multicolumn{4}{|l|}{ Deuterium $\left(\mathbf{D}_{2}\right)$} \\
\hline $1.5-30$ & $T, S$ & Hollricher, 1965 & $\mathrm{p}, \mathrm{y}$ \\
\hline \multicolumn{4}{|l|}{ Nitrogen $\left(\mathbf{N}_{2}\right)$} \\
\hline $150-1100$ & $T, S$ & Hooper, 1961 & $\mathrm{~d}, \mathrm{e}, \mathrm{g}, \mathrm{s}, \mathrm{t}, \mathrm{v}$ \\
\hline $15-180$ & $T, S$ & Solov'ev et al., 1962 & $c, j, r, t, v$ \\
\hline $1-40$ & $T, S$ & Gordeev and Panov, 1964 & $\mathrm{c}, \mathrm{j}, \mathrm{r}, \mathrm{t}, \mathrm{v}$ \\
\hline $10-140$ & $T, S$ & De Heer et al., 1966 & c \\
\hline $25-120$ & $T, S$ & Desesquelles et al., 1966 & $c, e, g, p, t, v$ \\
\hline $25-600$ & $T, S$ & Dufay et al., 1966 & $\mathrm{c}, \mathrm{e}, \mathrm{g}, \mathrm{p}, \mathrm{t}, \mathrm{v}$ \\
\hline
\end{tabular}


TABLE II. (Continued).

\begin{tabular}{|c|c|c|c|}
\hline $\begin{array}{c}\text { Energy } \\
(\mathrm{keV})\end{array}$ & Method $^{\mathrm{a}}$ & Investigator & Comments \\
\hline \multicolumn{4}{|c|}{ Diatomic targets } \\
\hline $1200-3000$ & $T, S$ & Pivovar and Levchenko, 1967 & ee \\
\hline $40-100$ & $T, S, M$ & Collins and Kebarle, 1967 & $\mathrm{~b}, \mathrm{c}, \mathrm{g}, \mathrm{p}, \mathrm{y}$ \\
\hline $2.5-20$ & $T, S$ & McNeal and Clark, 1969 & $\mathrm{c}, \mathrm{i}, \mathrm{p}, \mathrm{q}, \mathrm{t}, \mathrm{v}$ \\
\hline $20-120$ & $E$ & Schowengerdt and Park, 1970 & $\mathrm{jj}$ \\
\hline $200-500$ & $I, G$ & Stolterfoht, 1971 & cc,ii \\
\hline $50-300$ & $I$ & Crooks and Rudd, 1971 & \\
\hline 300 & $I$ & Toburen and Wilson, 1975 & $\mathrm{pp}$ \\
\hline $5-70$ & $I$ & Rudd, 1979 & \\
\hline $5-4000$ & $T, S$ & Rudd et al., 1983 & $\mathbf{u}$ \\
\hline \multicolumn{4}{|c|}{ Carbon Monoxide (CO) } \\
\hline $150-1100$ & $T, S$ & Hooper et al., 1961 & $\mathrm{~d}, \mathrm{e}, \mathrm{g}, \mathrm{s}, \mathrm{t}, \mathrm{v}$ \\
\hline $25-600$ & $T, S$ & Poulizac et al., 1966 & $c, e, g, p, t, v$ \\
\hline $25-100$ & $T, S$ & Desesquelles et al., 1966 & $c, e, g, p, t, v$ \\
\hline $25-620$ & $T, S$ & Poulizac et al., 1967 & $c, e, g, p, t, v$ \\
\hline $20-120$ & $E$ & Park et al., 1970 & $\mathrm{jj}$ \\
\hline $1-25$ & $T, S$ & McNeal, 1970 & $\mathrm{c}, \mathrm{i}, \mathrm{p}, \mathrm{q}, \mathrm{t}$ \\
\hline $5-4000$ & $T, S$ & Rudd et al., 1983 & $\mathrm{u}$ \\
\hline \multicolumn{4}{|l|}{ Oxygen $\left(\mathrm{O}_{2}\right)$} \\
\hline $150-1000$ & $T, S$ & Hooper, 1961 & $\mathrm{~d}, \mathrm{e}, \mathrm{g}, \mathrm{s}, \mathrm{t}, \mathrm{v}$ \\
\hline $10-140$ & $T, S$ & De Heer et al., 1966 & c \\
\hline $25-630$ & $T, S$ & Dufay et al., 1966 & $c, e, g, p, t, v$ \\
\hline $25-120$ & $T, S$ & Desesquelles et al., 1966 & $c, e, g, p, t, v$ \\
\hline $20-110$ & $E$ & Park et al., 1971 & $\mathrm{jj}$ \\
\hline $50-300$ & $I$ & Crooks and Rudd, 1971 & \\
\hline $2-20$ & $T, S$ & McNeal and Birely, 1973 & $f, o, x$ \\
\hline \multirow[t]{2}{*}{$5-4000$} & $T, S$ & Rudd et al., 1983 & $\mathbf{u}$ \\
\hline & \multicolumn{3}{|c|}{ Triatomic targets } \\
\hline \multicolumn{4}{|c|}{ Carbon Dioxide $\left(\mathrm{CO}_{2}\right)$} \\
\hline $25-120$ & $T, S$ & Desesquelles et al., 1966 & $\mathrm{c}, \mathrm{e}, \mathrm{g}, \mathrm{p}, \mathrm{t}, \mathrm{v}$ \\
\hline $25-600$ & $T, S$ & Poulizac and Dufay, 1967 & $c, e, g, p, t, v$ \\
\hline $1-25$ & $T, S$ & McNeal, 1970 & $\mathrm{c}, \mathrm{i}, \mathrm{p}, \mathrm{q}, \mathrm{t}$ \\
\hline $5-4000$ & $T, S$ & Rudd et al., 1983 & $\mathrm{u}$ \\
\hline \multicolumn{4}{|c|}{ Other targets } \\
\hline \multicolumn{4}{|l|}{ Ammonia $\left(\mathrm{NH}_{3}\right)$} \\
\hline $1-25$ & $T, S$ & McNeal, 1970 & $\mathrm{c}, \mathrm{i}, \mathrm{p}, \mathrm{q}, \mathrm{t}$ \\
\hline $250-2000$ & $I$ & Lynch et al., 1976 & $\mathrm{pp}$ \\
\hline \multicolumn{4}{|c|}{ Sulfur Hexafluoride $\left(\mathrm{SF}_{6}\right)$} \\
\hline $300-1800$ & $I$ & Toburen et al., 1977 & pp \\
\hline \multicolumn{4}{|c|}{ Tellurium Hexafluoride $\left(\mathrm{TeF}_{6}\right)$} \\
\hline $300-1800$ & $I$ & Toburen et al., 1977 & $\mathrm{pp}$ \\
\hline \multicolumn{4}{|l|}{ Methane $\left(\mathrm{CH}_{4}\right)$} \\
\hline $30-90$ & $T, S$ & Desesquelles et al., 1966 & $\mathrm{c}, \mathrm{e}, \mathrm{g}, \mathrm{p}, \mathrm{t}, \mathrm{v}$ \\
\hline $40-100$ & $T, S, M$ & Collins and Kebarle, 1967 & $\mathrm{~b}, \mathrm{c}, \mathrm{g}, \mathrm{p}, \mathrm{y}$ \\
\hline $1-25$ & $T, S$ & McNeal, 1970 & $\mathrm{c}, \mathrm{i}, \mathrm{p}, \mathrm{q}, \mathrm{t}$ \\
\hline $250-2000$ & $I$ & Lynch et al., 1976 & $\mathrm{pp}$ \\
\hline $10-25$ & $T, S$ & Mach et al., 1977 & $\mathrm{e}, \mathrm{o}, \mathrm{x}, \mathrm{y}$ \\
\hline $5-4000$ & $T, S$ & Rudd et al., 1983 & $\mathrm{u}$ \\
\hline \multicolumn{4}{|l|}{ Acetylene $\left(\mathrm{C}_{2} \mathrm{H}_{2}\right)$} \\
\hline $30-100$ & $T, S$ & Desesquelles et al., 1966 & $\mathrm{c}, \mathrm{e}, \mathrm{g}, \mathrm{p}, \mathrm{t}, \mathrm{v}$ \\
\hline \multicolumn{4}{|l|}{ Ethylene $\left(\mathrm{C}_{2} \mathrm{H}_{4}\right)$} \\
\hline $30-90$ & $T, S$ & Desesquelles et al., 1966 & $c, e, g, p, t, v$ \\
\hline $40-100$ & $T, S, M$ & Collins and Kebarle, 1967 & $\mathrm{~b}, \mathrm{c}, \mathrm{g}, \mathrm{p}, \mathrm{y}$ \\
\hline \multicolumn{4}{|l|}{ Ethane $\left(\mathrm{C}_{2} \mathrm{H}_{6}\right)$} \\
\hline $30-90$ & $T, S$ & Desesquelles et al., 1966 & $c, e, g, p, t, v$ \\
\hline \multicolumn{4}{|l|}{ Butane $\left(\mathrm{C}_{4} \mathrm{H}_{10}\right)$} \\
\hline $40-100$ & $T, S, M$ & Collins and Kebarle, 1967 & $\mathrm{~b}, \mathrm{c}, \mathrm{g}, \mathrm{p}, \mathrm{y}$ \\
\hline
\end{tabular}


TABLE II. (Continued).

\begin{tabular}{|c|c|c|c|}
\hline $\begin{array}{c}\text { Energy } \\
(\mathrm{keV})\end{array}$ & Method $^{\mathrm{a}}$ & Investigator & Comments \\
\hline \multicolumn{4}{|c|}{ Other targets } \\
\hline \multicolumn{4}{|l|}{ Monomethylamine $\left(\mathrm{CH}_{3} \mathrm{NH}_{2}\right)$} \\
\hline $250-2000$ & $I$ & Lynch et al., 1976 & $\mathrm{pp}$ \\
\hline \multicolumn{4}{|l|}{ Dimethylamine $\left[\left(\mathrm{CH}_{3}\right)_{2} \mathrm{NH}\right]$} \\
\hline $250-2000$ & $I$ & Lynch et al., 1976 & pp \\
\hline \multicolumn{4}{|l|}{ Methanol $\left(\mathrm{CH}_{3} \mathrm{OH}\right)$} \\
\hline $6-25$ & $T, S$ & Mach et al., 1977 & $e, o, x, y$ \\
\hline \multicolumn{4}{|l|}{ Ethanol $\left(\mathrm{C}_{2} \mathrm{H}_{5} \mathrm{OH}\right)$} \\
\hline $8-25$ & $T, S$ & Mach et al., 1977 & $e, o, x, y$ \\
\hline \multicolumn{4}{|l|}{ Propanol $\left(\mathrm{C}_{3} \mathrm{H}_{7} \mathrm{OH}\right)$} \\
\hline $8-25$ & $T, S$ & Mach et al., 1977 & $e, o, x, y$ \\
\hline \multicolumn{4}{|l|}{ Butanol $\left(\mathrm{C}_{4} \mathrm{H}_{9} \mathrm{OH}\right)$} \\
\hline $8-25$ & $T, S$ & Mach et al., 1977 & $e, o, x, y$ \\
\hline \multicolumn{4}{|l|}{ Pentanol $\left(\mathrm{C}_{5} \mathrm{H}_{11} \mathrm{OH}\right)$} \\
\hline $8-25$ & $T, S$ & Mach et al., 1977 & $e, o, x, y$ \\
\hline \multicolumn{4}{|l|}{ Hexanol $\left(\mathrm{C}_{6} \mathrm{H}_{13} \mathrm{OH}\right)$} \\
\hline $8-25$ & $T, S$ & Mach et al., 1977 & $e, o, x, y$ \\
\hline \multicolumn{4}{|l|}{ Heptanol $\left(\mathrm{C}_{7} \mathrm{H}_{15} \mathrm{OH}\right)$} \\
\hline $8-25$ & $T, S$ & Mach et al., 1977 & $e, o, x, y$ \\
\hline \multicolumn{4}{|l|}{ Octanol $\left(\mathrm{C}_{8} \mathrm{H}_{17} \mathrm{OH}\right)$} \\
\hline $8-25$ & $T, I$ & Mach et al., 1977 & $e, o, x, y$ \\
\hline \multicolumn{4}{|l|}{ Air } \\
\hline $5-180$ & $T, I$ & Il'in et al., 1959 & $\mathrm{c}, \mathrm{j}, \mathrm{r}, \mathrm{t}, \mathrm{v}$ \\
\hline
\end{tabular}

${ }^{a} T=$ transverse-field method, $E=$ energy-loss method, $I=$ method of integration of differential cross sections, $S=$ static-gas target, $\boldsymbol{G}=$ gas-beam target, $\boldsymbol{M}=$ mass and/or charge analysis of slow ions, $C=$ coincidence method.

${ }^{b}$ The proton beam was contaminated by $10 \% \mathrm{H}_{2}{ }^{+}$and possibly other heavier ions, since no magnetic analysis was used.

${ }^{\mathrm{c}}$ No correction was indicated for beam neutralization.

${ }^{\mathrm{d}}$ At low energies the beam may have been deflected by the transverse field so as to partially miss the Faraday cup.

' $T$ There is no indication that secondary electrons from the beam collimator were suppressed.

${ }^{f}$ Not enough information was given to judge the accuracy of the beam current measurement.

${ }^{\mathrm{g}} \mathrm{A}$ McLeod gauge was used without correcting for the Ishii effect.

${ }^{\text {h}}$ The pressure was measured by an ionization gauge calibrated by a McLeod gauge. No correction was indicated for the Ishii effect.

${ }^{i} A$ capacitance manometer was used with no correction indicated for thermal transpiration.

${ }^{\mathrm{j}}$ The pressure was measured with a radiometer-Knudsen gauge calibrated by a McLeod gauge. No correction was indicated for the Ishii effect.

${ }^{\mathrm{k}}$ The method of density measurement was not given, but was presumably by McLeod gauge without correction for the Ishii effect.

${ }^{1}$ Cross sections were obtained by normalization to previous data.

${ }^{\mathrm{m}}$ The target density was not measured. Cross sections were obtained by normalization to the Born approximation at high energy.

${ }^{\mathrm{n}}$ The pressure was measured with a Pirani gauge calibrated by a capacitance manometer. No correction was indicated for thermal transpiration.

${ }^{\circ}$ Not enough information was given to judge the accuracy of the density determination.

${ }^{\mathrm{P}} \mathrm{Guard}$ plates were not used or did not appear to have been large enough.

"Saturation of the electron current was probably not achieved below $3 \mathrm{keV}$ since the transverse field had to be reduced to avoid beam deflection.

${ }^{\mathrm{r}}$ A longitudinal field from the Faraday cup may have attracted electrons away from the measurement region.

${ }^{\text {s }}$ The large collecting plate potentials used may have caused appreciable leakage currents.

${ }^{t}$ No correction was indicated for the difference between the geometric transmission of the grid and its transmission for charged particles.

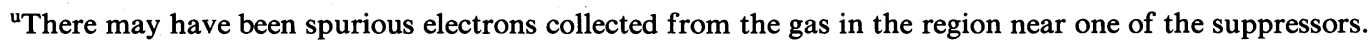

${ }^{v}$ No correction was indicated for the secondary electrons produced when positive ions strike the grid.

${ }^{w}$ The electron current was not measured. The cross section $\sigma_{-}$was obtained by subtracting $\sigma_{c}$ from $\sigma_{+}$.

${ }^{x}$ Not enough information was given to judge the accuracy of the electron current measurement.

${ }^{\mathrm{y}}$ The secondary electrons from slow-ion impact were not suppressed.

${ }^{\mathrm{z}}$ The electron current was not measured. The cross section $\sigma_{-}$was assumed to be equal to $\sigma_{+}$.

${ }^{\text {aa }}$ The cross sections were normalized to those of Hooper (1961) at each proton energy.

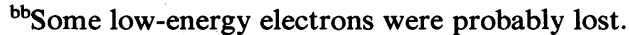

${ }^{c c}$ The pre-acceleration potential may have distorted the collection geometry for low-energy electrons.

${ }^{\mathrm{dd}}$ No grid was used, but an analysis of current sources using a different electrode system allowed subtraction of secondary current. 
TABLE II. (Continued).

${ }^{e}$ The pressure was measured with a McLeod gauge cooled to $0^{\circ} \mathrm{C}$.

${ }^{\text {ff }}$ The Faraday cup was negatively biased, allowing secondary electrons to escape.

ggThe data were normalized to the cross-section data of Schwirzke (1960) at $50 \mathrm{keV}$.

${ }^{\mathrm{h} h}$ The determination of the detector efficiency was probably not accurate.

${ }^{i}$ Low-energy secondary electrons from surfaces may have been collected.

${ }^{i j A t}$ low energies some protons may have been scattered outside the angular acceptance of the energy-loss analyzer.

${ }^{\mathrm{kk}}$ Data were normalized to the Born approximation cross section for excitation to the $n=2$ state of hydrogen.

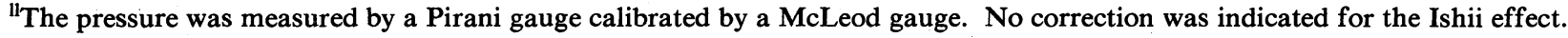

${ }^{\mathrm{mm}}$ The pressure was measured by an ionization gauge. No information was given to indicate how it was calibrated.

${ }^{n n}$ The data were normalized to the capture cross sections of Steddeford and Hasted (1955).

${ }^{\infty}$ No indication of the method of measuring the electron current was given.

${ }^{\mathrm{PP}}$ The density distribution of the target gas varied with angle, requiring corrections.

${ }^{\mathrm{qq}}$ The target density was not measured. Cross sections were obtained by normalization to electron capture data of Stier and Barnett (1956).

indicates the presence of systematic errors either not known or not fully appreciated by the authors. To deal with such discrepancies, the following procedures were adopted for this review.

(1) If an error could be identified and its magnitude calculated, then the cross sections were corrected accordingly. This was seldom possible because dimensions, pressures, and other information needed to make these corrections are not often given in published reports. Authors tend to describe their measurements in the best light and fail to point out possible sources of error and inconsistencies. In some cases, descriptions of the apparatus are omitted entirely. Thus it is necessary to deal with data containing unknown amounts of systematic errors.

(2) Next, each experiment was evaluated on the basis of the published account. An estimate of the probable size of the systematic error was made based on three factors. These were (a) the author's own estimate of the uncertainty in his experiment, (b) our own estimate of the error based on our judgment of the care with which the experiment was done, the corrections that were made, and the attention to various experimental criteria already discussed, and (c) the extent of disagreement with the average of other experimental values. Clearly, factor (b) is a highly subjective process but a necessary one, because all data are not of equal quality. The errors assigned in (a), (b), and (c) were averaged to determine the weighting of a data set in the fitting process.

(3) The data available for each target were averaged by assigning a weight to each data set and then making a least-squares fit of a mathematical equation to the data.

\section{B. Weighting the data}

Mathematical methods for dealing with independent data with random errors are well developed, but the problems of how to deal with systematic errors are seldom addressed. In most of the data sets encountered in this study, the systematic error is considerably greater than the random error. This is indicated by the fact that the fluctuations of a given set from point to point are usually smaller than the discrepancies among authors, especially for lower proton energies.

To approach this problem, a weight that is the product of three weighting factors was determined for each cross section. The first is the weight factor (see, for example, Bevington, 1969) for random errors in independent data, which is the reciprocal of the square of the absolute error

$$
W_{1}=1 /(\delta \sigma)^{2} \text {, }
$$

where $\delta$ is the relative error and $\sigma$ is the value of the measured quantity, in this case the cross section. The relative error was assigned as described in Sec. VI.A.

In using this equation the fact that the systematic error is not random is ignored. But what about the requirement that the data be independent? Other things being equal, an equal weight should be assigned to all independent measurements, But what constitutes an "independent" measurement? The various cross sections given by one author are not really independent, since they were all taken on the same apparatus using the same method and thus have mostly the same systematic errors. An author, for example, who measures a cross section at a given energy 50 times may reduce his random error by the large number of measurements, but his systematic error remains. Since this is usually the more important error, his data should not get a 10 times greater weight than that of another author who measured the value only 5 times. However, this would be the result of weighting every measurement equally. Therefore each data set by an author is treated here as an independent measurement, and the weight assigned to that set is divided by $N$, the number of points in the set, providing the second factor in the weight $W_{2}=1 / N$.

Because the systematic error tends to change as a function of energy, data from the same set taken at different energies are at least partially independent. This is recognized by including a third weighting factor,

$$
W_{3}=\log _{10}\left(1+E_{\max } / E_{\min }\right),
$$

where $E_{\max }$ and $E_{\min }$ are the maximum and minimum values of the energy range covered in the data set. While the form of this factor cannot be defended in detail, it seems generally reasonable. This factor gives a relative 
weight, e.g., of 0.3 to a cross section at a single energy, a weight of approximately 1 for data taken over an energy range of 10 to 1,2 for a range of 100 to 1 , etc.

Thus the overall weight given to each data point in a set of $N$ such points is the product of the three weighting factors,

$W=W_{1} W_{2} W_{3}=\left(1 / N \delta^{2} \sigma^{2}\right) \log _{10}\left(1+E_{\max } / E_{\min }\right)$.

\section{Fitting equations}

Because the various data sets cover different energy ranges, the elementary procedure of averaging the values at each energy would lead to discontinuities in the resulting energy dependence of the cross sections. Langenberg and van Eck (1976) approached this problem for $K$-shell ionization data by multiplying the data in each set by a constant to bring it into line with the weighted mean of all the data sets. For this method to work, however, the energy dependence of all the data sets must be the same. Significant differences exist in shapes among the data sets considered here. Therefore, a fit was made to a mathematical equation for all of the data after assigning a weight to each data set. The criteria by which the fitting equation was chosen were as follows.

(1) The equation should have the correct high-energy behavior, which is assumed to be the energy dependence predicted by the Bethe approximation,

$$
\sigma \propto[A \ln (1+x)+B] / x, \text { where } x=T / R .
$$

(2) It should have a reasonable low-energy behavior, which is assumed to be a power-law dependence on the energy.

(3) The equation should be relatively simple.

(4) It should have a small number of adjustable parameters.

(5) The same form of the equation should be usable for all targets.

(6) It should fit the energy dependence of the data within the experimental uncertainty.

There have been many empirical equations proposed to fit electron-impact ionization data, some of which were reviewed by Drawin (1961), but few equations have been given for proton-impact ionization.

With different values of the parameters, Eq. (24) holds for electron-impact ionization. At high energies, this becomes $\sigma \propto T^{-1} \ln T$. Bethe's treatment also shows that for protons in the asymptotic energy region the cross sections should be exactly the same as for electrons, provided that the comparison is made at the same velocity. The two cases can be conveniently compared by defining

$$
T=m_{e} v^{2} / 2=E_{p} / 1836,
$$

where $v$ and $E_{p}$ are the velocity and energy of the proton, respectively. By defining $T$ in this way, any of the electron equations can be used for high-energy protons as well.

At low energy two differences from electron-impact ionization are encountered. First, while most of the empirical equations for electron-impact ionization assume that the cross section goes as the first power of the excess of the energy above threshold, for protons the value of the power may differ from unity. Second, while the threshold for electrons comes at $T=I$, where $I$ is the ionization potential, for protons the threshold is $E_{p}=I$ or $T=I / 1836$. Thus we shall assume the low-energy cross section to be of the form $\left(E_{p}-I\right)^{D}$, where $D$ is to be determined for each target by fitting. Since for all the experiments reviewed here $E_{p} \gg I$, we shall drop the $I$.

Several empirical equations, each of which gives the desired low-energy and asymptotic dependences, were tried. The one chosen is a simple combination of the cross sections appropriate to the high- and low-energy regions,

$$
\sigma_{-}=\left(\sigma_{l}^{-1}+\sigma_{h}^{-1}\right)^{-1}
$$

where

$$
\sigma_{l}=4 \pi a_{0}^{2} C x^{D}
$$

and

$$
\sigma_{h}=4 \pi a_{0}^{2}[A \ln (1+x)+B] / x,
$$

with $x=T / R, a_{0}=0.529 \AA$, and $R=13.6 \mathrm{eV} . A, B, C$, and $D$ are the adjustable fitting parameters.

The asymptotic behavior of the fitting equation differs from the first two terms of the Bethe equation only by the addition of unity in the argument of the logarithm. Therefore the parameter $A$ can be compared directly to the corresponding value obtained by electron impact and by photoionization after appropriate adjustments for differences in multiple ionization. The quantity $B$ is affected by the rest of the equation and is not directly comparable to the corresponding quantity in the Bethe equation. Furthermore, in the fitting process $B$ had to be restricted to positive values or zero, because the equation is applied over the entire energy range; if $B$ were negative it would cause $\sigma_{h}$ to go negative at some value of energy.

Because the systematic error in these measurements seems to exceed the random error, the small cross sections at the highest energies usually have a fractional error no greater than that of the larger cross sections at intermediate energies. An ordinary least-squares fit would, however, give the smaller cross sections less weight, due to their smaller absolute values. Therefore, instead of minimizing the squares of the absolute deviations, the fractional deviations were used.

\section{Recommended values}

After finding the parameters of Eqs. (32) and (33) that give the best fit to the experimental data for each target, the results were compared to theoretical values, to the data from photoionization, and to electron-impact data. For the photoionization data the values of the optical oscillator strength compiled by Berkowitz (1979), specifically $S_{i}(-1)$ in his notation, were used. On the basis of this 
TABLE III. Values of fitting parameters for Eqs. (32) and (33).

\begin{tabular}{cccccccc}
\hline \hline Target & $A$ & $B$ & $C$ & $D$ & Low $E$ & $\begin{array}{c}\text { Reliability }^{\text {a }} \\
\text { Near max }\end{array}$ & High $E$ \\
\hline $\mathrm{H}$ & 0.28 & 1.15 & 0.44 & 0.907 & $c$ & $b$ & $a$ \\
$\mathrm{He}$ & 0.49 & 0.62 & 0.13 & 1.52 & $c$ & $b$ & $a$ \\
$\mathrm{Ne}$ & 1.63 & 0.73 & 0.31 & 1.14 & $c$ & $b$ & $a$ \\
$\mathrm{Ar}$ & 3.85 & 1.98 & 1.89 & 0.89 & $c$ & $c$ & $b$ \\
$\mathrm{Kr}$ & 5.67 & 5.50 & 2.42 & 0.65 & $c$ & $c$ & $b$ \\
$\mathrm{Xe}$ & 7.33 & 11.1 & 4.12 & 0.41 & $c$ & $b$ & $b$ \\
$\mathrm{H}_{2}$ & 0.71 & 1.63 & 0.51 & 1.24 & $c$ & $b$ & $a$ \\
$\mathrm{~N}_{2}$ & 3.82 & 2.78 & 1.80 & 0.70 & $b$ & $b$ & $a$ \\
$\mathrm{O}_{2}$ & 4.77 & 0.00 & 1.76 & 0.93 & $c$ & $b$ & $a$ \\
$\mathrm{CO}$ & 3.67 & 2.79 & 2.08 & 1.05 & $b$ & $b$ & $b$ \\
$\mathrm{CO}$ & 6.55 & 0.00 & 3.74 & 1.16 & $b$ & $c$ & $b$ \\
$\mathrm{NH}_{3}$ & 4.01 & 0.00 & 1.73 & 1.02 & $c$ & $c$ & $b$ \\
$\mathrm{CH}_{4}$ & 4.55 & 2.07 & 2.54 & 1.08 & $c$ & $b$ & $b$ \\
\hline \hline
\end{tabular}

${ }^{\mathrm{a}} a:<10 \%$. b: 10-25\%. c: $>25 \%$.

comparison, some adjustments to one or more parameters were made and the equation was refitted. This generally did not affect the fit very much, but did improve the consistency with photoionization data at high energies.

Table III shows the recommended values of the four parameters of Eqs. (32) and (33) for each target. Also the estimated reliabilities of the fitted values for three different energy ranges are presented. Values of the cross sections calculated from Eq. (31) are given in Table IV.

Each case is now discussed individually.

\section{Atomic hydrogen}

The initial fitted value of $A=0.27$ agreed very well with the theoretical value of 0.28 . In this case the wave functions are well known and complications such as multiple ionization or inner-shell effects are not present. As a result, the theoretical methods described above should be very accurate at high energy. Consequently the slight adjustment of $A$ to the theoretical value was made, and the other parameters varied to fit the data. The resulting

TABLE IV. Values of recommended cross sections $\left(10^{-20} \mathrm{~m}^{2}\right)$.

\begin{tabular}{|c|c|c|c|c|c|c|c|c|c|c|c|c|c|}
\hline $\begin{array}{c}\text { Energy } \\
(\mathrm{keV})\end{array}$ & $\mathbf{H}$ & $\mathrm{He}$ & $\mathrm{Ne}$ & Ar & $\mathrm{Kr}$ & $\mathrm{Xe}$ & $\mathbf{H}_{2}$ & $\mathbf{N}_{2}$ & $\mathrm{O}_{2}$ & $\mathrm{CO}$ & $\mathrm{CO}_{2}$ & $\mathrm{NH}_{3}$ & $\mathrm{CH}_{4}$ \\
\hline 0.3 & & & 0.00705 & 0.130 & & & & & & & & & \\
\hline 0.5 & & 0.00118 & 0.0126 & 0.204 & & & 0.0140 & & & & 0.140 & & 0.134 \\
\hline 0.7 & & 0.00197 & 0.0185 & 0.276 & & & 0.0213 & & & & 0.206 & & 0.192 \\
\hline 1.0 & 0.084 & 0.00338 & 0.0278 & 0.378 & 1.05 & 3.86 & 0.0332 & 0.661 & 0.305 & 0.249 & 0.310 & & 0.281 \\
\hline 1.5 & 0.122 & 0.00626 & 0.0441 & 0.542 & 1.37 & 4.54 & 0.0548 & 0.876 & 0.441 & 0.380 & 0.491 & & 0.434 \\
\hline 2.0 & 0.158 & 0.00969 & 0.0612 & 0.698 & 1.64 & 5.10 & 0.0782 & 1.07 & 0.571 & 0.514 & 0.680 & 0.447 & 0.589 \\
\hline 3.0 & 0.227 & 0.0179 & 0.0969 & 0.994 & 2.13 & 5.98 & 0.129 & 1.41 & 0.818 & 0.783 & 1.07 & 0.664 & 0.905 \\
\hline 5.0 & 0.358 & 0.0389 & 0.172 & 1.54 & 2.92 & 7.25 & 0.242 & 1.98 & 1.27 & 1.32 & 1.85 & 1.08 & 1.54 \\
\hline 7.0 & 0.478 & 0.0646 & 0.251 & 2.02 & 3.58 & 8.17 & 0.364 & 2.45 & 1.68 & 1.84 & 2.61 & 1.46 & 2.16 \\
\hline 10 & 0.642 & 0.110 & 0.371 & 2.67 & 4.39 & 9.19 & 0.557 & 3.04 & 2.23 & 2.59 & 3.68 & 1.99 & 3.02 \\
\hline 15 & 0.871 & 0.199 & 0.570 & 3.55 & 5.43 & 10.3 & 0.880 & 3.79 & 2.98 & 3.68 & 5.18 & 2.72 & 4.27 \\
\hline 20 & 1.05 & 0.297 & 0.759 & 4.22 & 6.21 & 11.1 & 1.18 & 4.34 & 3.58 & 4.57 & 6.34 & 3.30 & 5.27 \\
\hline 30 & 1.26 & 0.494 & 1.09 & 5.11 & 7.24 & 11.9 & 1.67 & 5.04 & 4.41 & 5.78 & 7.82 & 4.09 & 6.57 \\
\hline 50 & 1.37 & 0.789 & 1.56 & 5.79 & 8.13 & 12.3 & 2.12 & 5.58 & 5.18 & 6.69 & 8.83 & 4.76 & 7.49 \\
\hline 70 & 1.29 & 0.916 & 1.80 & 5.82 & 8.28 & 12.0 & 2.16 & 5.61 & 5.35 & 6.67 & 8.75 & 4.85 & 7.43 \\
\hline 100 & 1.12 & 0.926 & 1.90 & 5.47 & 7.99 & 11.3 & 1.96 & 5.33 & 5.20 & 6.14 & 8.11 & 4.62 & 6.84 \\
\hline 150 & 0.882 & 0.810 & 1.80 & 4.75 & 7.17 & 9.98 & 1.60 & 4.70 & 4.67 & 5.19 & 6.96 & 4.08 & 5.80 \\
\hline 200 & 0.721 & 0.695 & 1.63 & 4.14 & 6.39 & 8.86 & 1.33 & 4.15 & 4.17 & 4.44 & 6.06 & 3.60 & 4.99 \\
\hline 300 & 0.528 & 0.533 & 1.34 & 3.29 & 5.18 & 7.21 & 0.988 & 3.34 & 3.40 & 3.45 & 4.82 & 2.90 & 3.91 \\
\hline 500 & 0.347 & 0.367 & 0.973 & 2.35 & 3.76 & 5.26 & 0.662 & 2.41 & 2.49 & 2.42 & 3.47 & 2.11 & 2.77 \\
\hline 700 & 0.261 & 0.283 & 0.770 & 1.85 & 2.97 & 4.17 & 0.504 & 1.90 & 1.99 & 1.89 & 2.75 & 1.68 & 2.17 \\
\hline 1000 & 0.192 & 0.214 & 0.592 & 1.42 & 2.28 & 3.21 & 0.375 & 1.46 & 1.55 & 1.44 & 2.13 & 1.30 & 1.66 \\
\hline 1500 & 0.134 & 0.154 & 0.434 & 1.04 & 1.67 & 2.35 & 0.267 & 1.07 & 1.15 & 1.04 & 1.57 & 0.964 & 1.22 \\
\hline 2000 & - & 0.121 & 0.347 & 0.829 & 1.33 & 1.87 & 0.209 & 0.853 & 0.920 & 0.830 & 1.26 & 0.773 & 0.970 \\
\hline 3000 & & 0.0868 & 0.251 & 0.599 & 0.956 & 1.34 & 0.148 & 0.615 & 0.671 & 0.597 & 0.919 & 0.563 & 0.700 \\
\hline 5000 & & 0.0564 & 0.165 & 0.394 & 0.626 & & 0.0950 & 0.404 & 0.445 & 0.391 & 0.610 & 0.374 & 0.461 \\
\hline
\end{tabular}




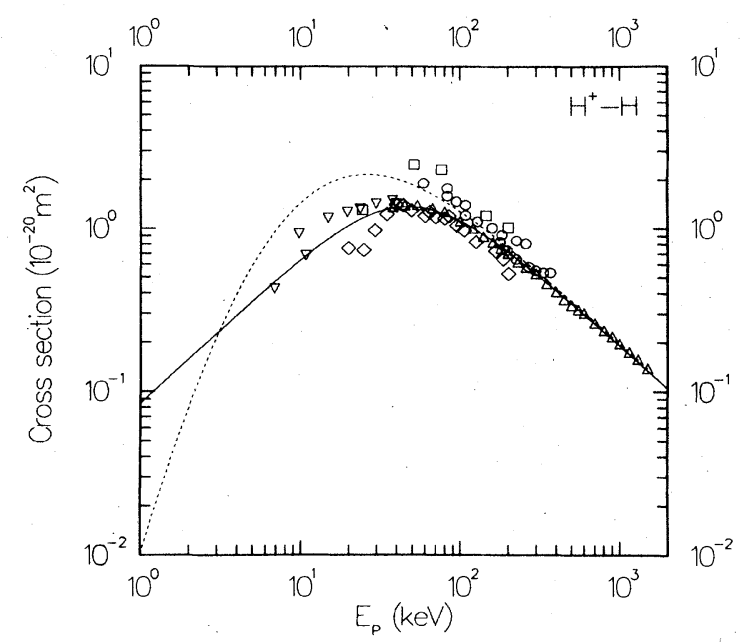

FIG. 4. Cross sections for ejection of electrons in $\mathrm{H}^{+}+\mathrm{H}$ collisions as a function of energy. The solid curve is the recommended fit; the dashed curve is the DWBA calculation. Experimental data: $\nabla$, Fite (1960); $\bigcirc$, Gilbody and Ireland (1963); $\square$, Park et al. (1977); $\triangle$, Shah and Gilbody (1981); $\diamond$, Park (1983).

curve shown in Fig. 4 and on the Fano plot of Fig. 5 is in good agreement with the experimental data at high energy, especially that of Shah and Gilbody (1981), which is the most accurate for this target. At low energies, however, the data are less reliable and the uncertainties greater.

\section{Helium}

As shown in Fig. 6, the fit of Eq. (31) to the experimental data for helium is in excellent agreement with the

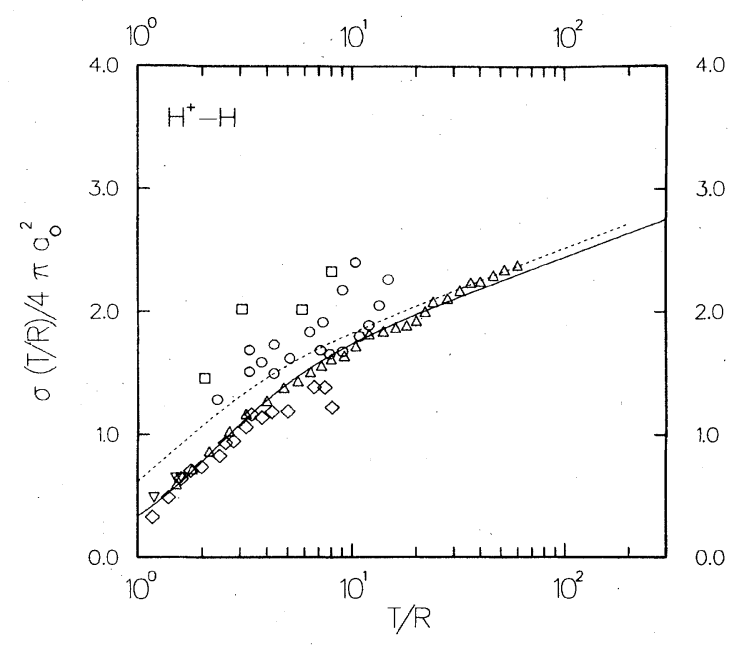

FIG. 5. Fano plot for ionization of $\mathrm{H}$ atoms by $\mathrm{H}^{+} . \quad T$ is the scaled energy, $T=E_{p} / 1836, R$ is the Rydberg energy, and $a_{0}$ is the Bohr radius. The solid curve is the recommended fit; the dashed curve is the DWBA calculation. Experimental data points: $\nabla$, Fite (1960); $\bigcirc$, Gilbody and Ireland (1963); $\square$, Park et al. (1977); $\triangle$, Shah and Gilbody (1981); $\diamond$, Park (1983).

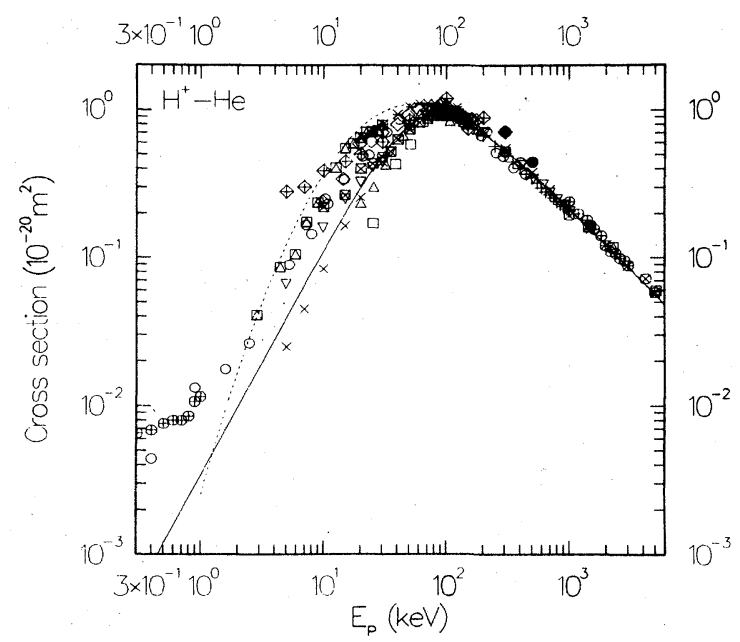

FIG. 6. Cross sections for ejection of electrons in $\mathrm{H}^{+}+\mathrm{He}$ collisions as a function of energy. The solid curve is the recommended fit. The dashed curve is the DWBA calculation. Experimental data points: $\nabla$, Keene (1949); $O$, Gilbody and Hasted (1957) and Gilbody and Lee (1963); $\triangle$, Fedorenko et al. (1960); +, Hooper (1961); $\diamond$, Solov'ev et al. (1962); \& , Rudd and Jorgensen (1963); $\bigotimes$, De Heer et al. (1966); $*$, Desesquelles et al. (1966); $\oplus$, Rudd et al. (1966) and Rudd and Madison (1976); $\bigoplus\left(E_{p} \geq 1000 \mathrm{keV}\right)$, Pivovar and Levchenko (1967); $\nabla$ $\left(E_{p} \leq 100 \mathrm{keV}\right)$, Becker and Scharmann (1969); $\nabla$, Puckett and Martin (1970); ๑, Stolterfoht (1971a); $\bigoplus\left(E_{p}<11 \mathrm{keV}\right)$, Latypov and Shaporenko (1973); $\otimes$, Toburen, from Rudd et al. (1976); $\bigotimes$, Manson et al., from Rudd et al. (1976); $\nabla\left(E_{p}>1000 \mathrm{keV}\right)$, Hvelplund et al. (1980); $\times$, Rudd et al. (1983).

DWBA at high energies. It also agrees very well with the electron data (see Fig. 7) and with optical data, and therefore no adjustment was needed. As mentioned earlier, proton- and electron-impact data are expected to ap-

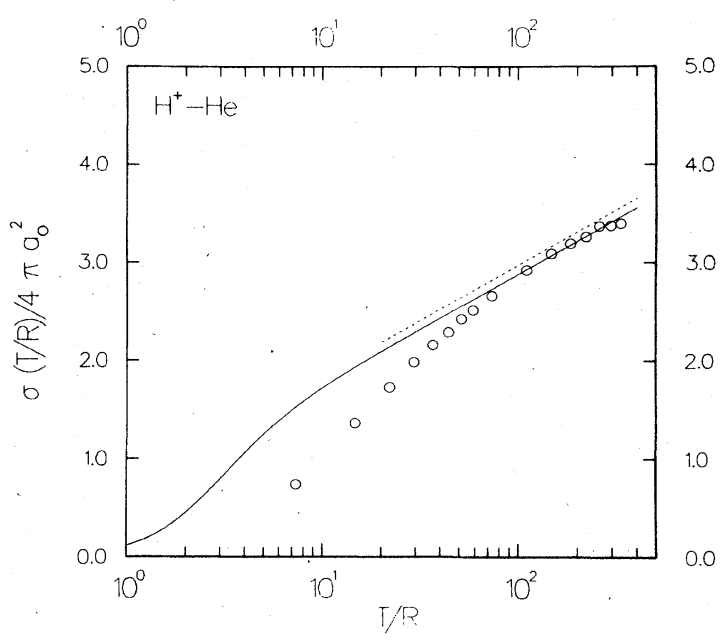

FIG. 7. Fano plot for ionization of He. $T$ is the scaled energy, $T=E_{p} / 1836, R$ is the Rydberg energy, and $a_{0}$ is the Bohr radius. The solid curve is the recommended proton-impact ionization cross section, the dashed curve is the Bethe cross section for electron-impact ionization (Kim and Inokuti, 1971), and the circles represent electron-impact experimental values of Smith (1930). 
proach each other at high incident energies (at equal velocities) for targets with simple electronic structure. Figure 7 shows that this is indeed the case for $E_{p}>1 \mathrm{MeV}$ $(T>500 \mathrm{eV})$. Among the many electron-impact data on He, the experimental data by Smith (1930) are shown because they agree best with theory (Kim and Inokuti, 1971) at high energies. At low energies there is a very large spread in the proton data. This is probably due in part to the small size of the helium cross sections, which magnifies the problems of spurious currents and the errors in beam collection. Since both of these tend to cause the measured cross sections to be too large, the largest measured values should be the least reliable and were given less weight.

\section{Neon}

From the data of DuBois, Toburen, and Rudd (1984) on multiple ionization, one can show that at high energies the ratio, $\kappa_{-}$[see Eq. (8)] is 1.015 . Therefore $A$ was set to be $1.5 \%$ greater than the optical value of 1.61 , and Eqs. (32) and (33) were refitted accordingly. The resulting fit is slightly below the DWBA value, although at high energies the experimental cross sections are expected to be $1.5 \%$ higher than the calculated values. Figure 8 shows the results. There is a large disagreement at low energies between the data of Gilbody and Hasted (1957) and those of Latypov and Shaporenko (1973). In spite of the innovative approach in the latter set of data (see Sec. IV.A.7), this data set is not as reliable and therefore was given less weight.

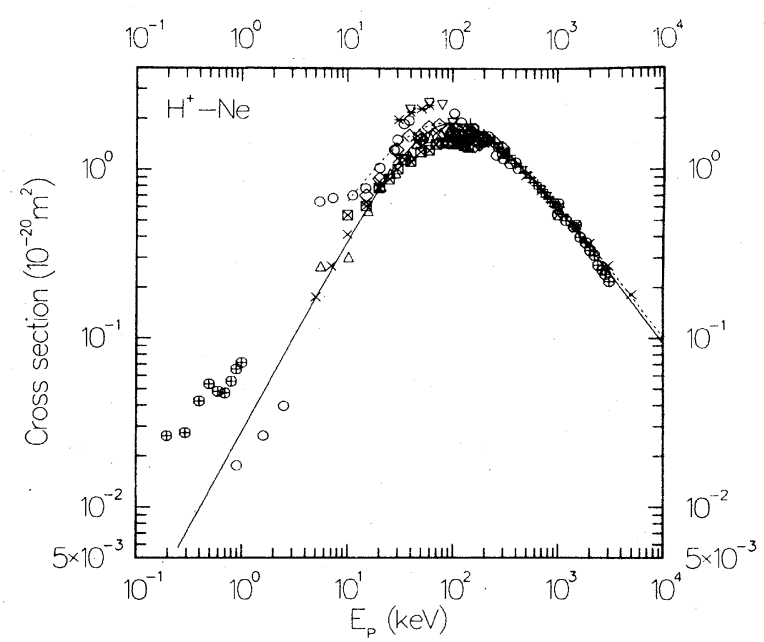

FIG. 8. Cross sections for ejection of electrons in $\mathrm{H}^{+}+\mathrm{Ne}$ collisions as a function of energy. The solid curve is the recommended fit; the dashed curve is the DWBA calculation. Experimental data: O, Gilbody and Hasted (1957) and Gilbody and Lee (1963); $\triangle$, Fedorenko et al. (1960); + , Hooper et al. (1961); $\diamond$, Solov'ev et al. (1962); $\bigotimes$, De Heer et al. (1966); $*$, Desesquelles et al. (1966); $\bigoplus\left(E_{p} \geq 1000 \mathrm{keV}\right)$, Pivovar and Levchenko (1967); $\nabla$, Collins and Kebarle (1967); $\oplus$, Crooks and Rudd (1971); $\bigoplus\left(E_{p} \leq 1 \mathrm{keV}\right)$, Latypov and Shaporenko (1972); $\bigotimes$, Toburen et al. (1978); $\times$, Rudd et al. (1983).

\section{Argon}

The parameter $A$ from the weighted fit was 4.51, considerably larger than the optical value of 3.53. From the data of DuBois, Toburen, and Rudd (1984) on multiple ionization, the value of $\kappa_{-}$was found to be about 1.09 at high energies. Therefore $A$ was taken to be $9 \%$ larger than the optical value. Even though this is still $15 \%$ below the value from fitting, the resulting curve (see Fig. 9) still fits the data quite well. Again the results are lower than those given by the DWBA, but these theoretical values are not expected to be reliable for atorns as large as Ar.

\section{Krypton}

The value of $A$ obtained from the fit is 5.67 , which is about $24 \%$ higher than the optical value of 4.57. Actually, according to the multiple-ionization data of DuBois, Toburen, and Rudd (1984), the value should be about $40 \%$ higher than the optical value. Because of the uncertainties in this correction for heavier atoms, the unadjusted weighted fit for krypton was retained. The results are shown in Fig. 10.

\section{Xenon}

The parameters from the weighted fit were retained without adjustment. While it would be expected that

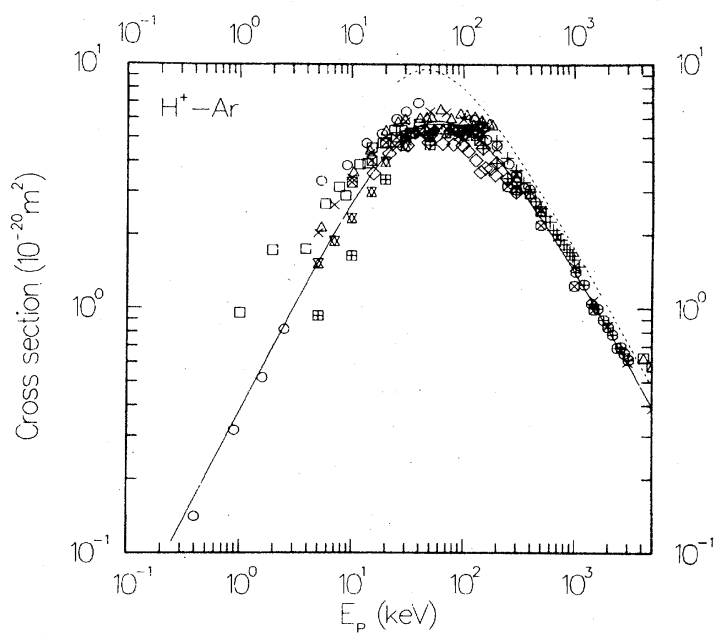

FIG. 9. Cross sections for ejection of electrons in $\mathrm{H}^{+}+\mathrm{Ar}$ collisions as a function of energy. The solid curve is the recommended fit; the dashed curve is the DWBA calculation. Experimental data: O, Gilbody and Hasted (1957) and Gilbody and Lee (1963); $\triangle$, Fedorenko et al. (1960); +, Hooper et al. (1961); $\diamond$, Solov'ev et al. (1962); $\square$, Gordeev and Panov (1964); $\bigotimes$, De Heer et al. (1966); $\star$, Desesquelles et al. (1966); $\bigoplus$, Pivovar and Levchenko (1967); $\oplus$, Crooks and Rudd (1971); $\triangle$, Gabler, from Rudd et al. (1979); $\boxplus$, Criswell et al. (1977); $\&$, Rudd, from Criswell et al. (1977); $\bigotimes$, Toburen et al. (1978); $\times$, Rudd et al. (1983). 


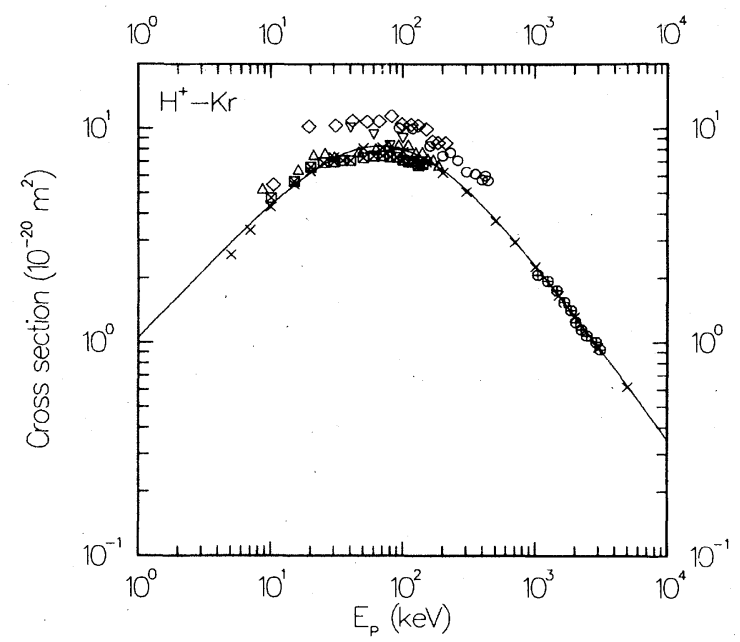

FIG. 10. Cross sections for ejection of electrons in $\mathrm{H}^{+}+\mathrm{Kr}$ collisions as a function of energy. The solid curve is the recommended fit. Experimental data: $\triangle$, Fedorenko et al. (1960); $\diamond$, Solov'ev et al. (1962); O, Gilbody and Lee (1963); $\bigotimes$, De Heer et al. (1966); $\bigoplus$, Pivovar and Levchenko (1967); $\nabla$, Collins and Kebarle (1967); $\times$, Rudd et al. (1983).

multiple ionization would make the value of $A$ from the proton data larger than the optical value, the value obtained from the fitting is 6.00 while the optical value is 6.12. The small amount of data available appears to be reliable, but does not extend to very low energies. The results are shown in Fig. 11.

\section{Molecular hydrogen}

There is a considerable spread in the high-energy data for $\mathrm{H}_{2}$, as shown in the Fano plot of Fig. 12. The data of

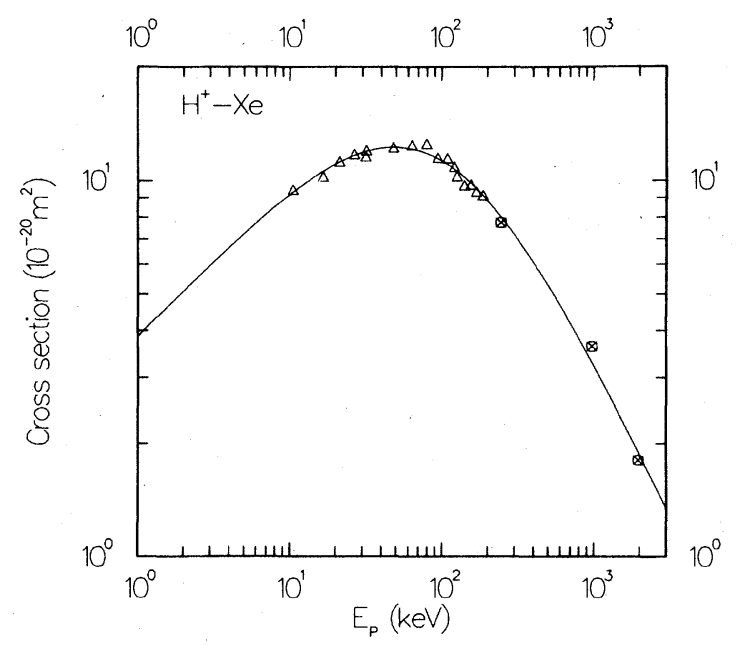

FIG. 11. Cross sections for ejection of electrons in $\mathrm{H}^{+}+\mathrm{Xe}$ collisions. The solid curve is the recommended fit. Experimental data: $\triangle$, Fedorenko et al. (1960); $\bigotimes$, Toburen (1974).

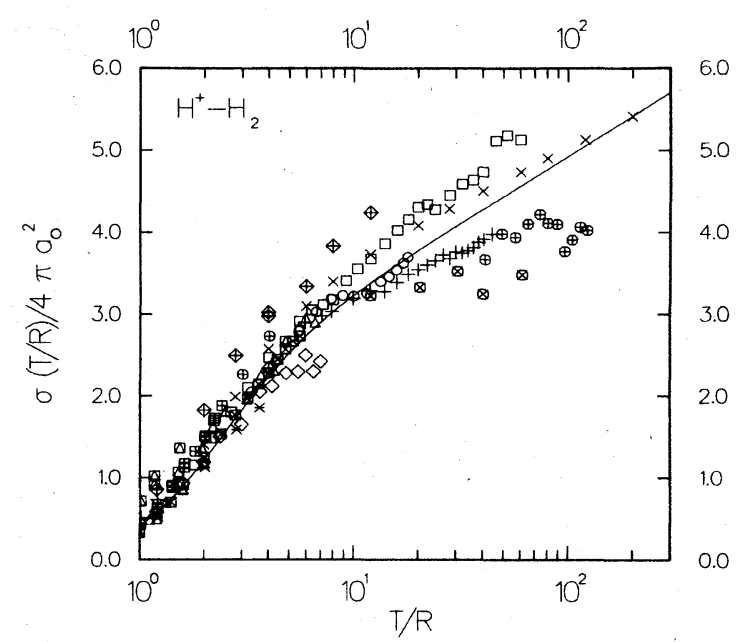

FIG. 12. Fano plot for ionization of $\mathrm{H}_{2}$ molecules by $\mathrm{H}^{+}$. The solid curve is the recommended fit. Experimental data: $\triangle$, Afrosimov et al. (1958a); $\boxplus$, Schwirzke (1960); $+\left(E_{p}>100\right.$ $\mathrm{keV}$ ), Hooper et al. (1961); $\diamond$, Solov'ev et al. (1962); $\oplus$ $\left(E_{p} \leq 100 \mathrm{keV}\right)$, Kuyatt and Jorgenson (1963); $\oplus$, Rudd and Jorgensen (1963), Rudd et al. (1966) and Rudd (1979); O, Gilbody and Lee (1963); $\triangle$, Gordeev and Panov (1964); + $\left(E_{p}<100\right.$ $\mathrm{keV}$ ), Hollricher (1965); $\bigotimes$, De Heer et al. (1966); $*$, Desesquelles et al. (1966); $\oplus\left(E_{p} \geq 1000 \mathrm{keV}\right)$, Pivovar and Levchenko (1967); $\triangle$, Afrosimov et al. (1969); $\bigotimes$, Toburen and Wilson (1972); $\square$, Shah and Gilbody (1982); $\times$, Rudd et al. (1983).

Shah and Gilbody (1982) favor an $A$ value of about 0.8 , while the data of Hooper (1961) and of Rudd et al. (1983) yield $A$ values of about 0.6 . Therefore the optical value of 0.71 was used here and the other three parameters adjusted to fit the data. While the available electron data do not go to a high enough energy to confirm this number, they are at least consistent with it. The data of De Heer et al. (1966) and of Shah and Gilbody (1982) were given the most weight at high energies, and that of Rudd et al. (1983) was assumed to be the most reliable at low energies. The data and fit are shown in Fig. 13.

\section{Nitrogen}

The parameters from the fitting have been retained without adjustment. The $A$ value of 3.82 is in fairly good agreement with the optical value of 3.43 , when allowance is made for dissociative ionization, which is likely to be greater for proton ionization than for photoionization. The data sets of De Heer et al. (1966) and of Rudd et al. (1983) were given the most weight. Figure 14 shows the results for nitrogen.

In Fig. 15 our fitted cross section is compared with electron-impact data of Rapp and Englander-Golden (1965) and a semiempirical cross section adopted by Kim (1975b) for fast incident electrons. As in the case of $\mathrm{He}$, 


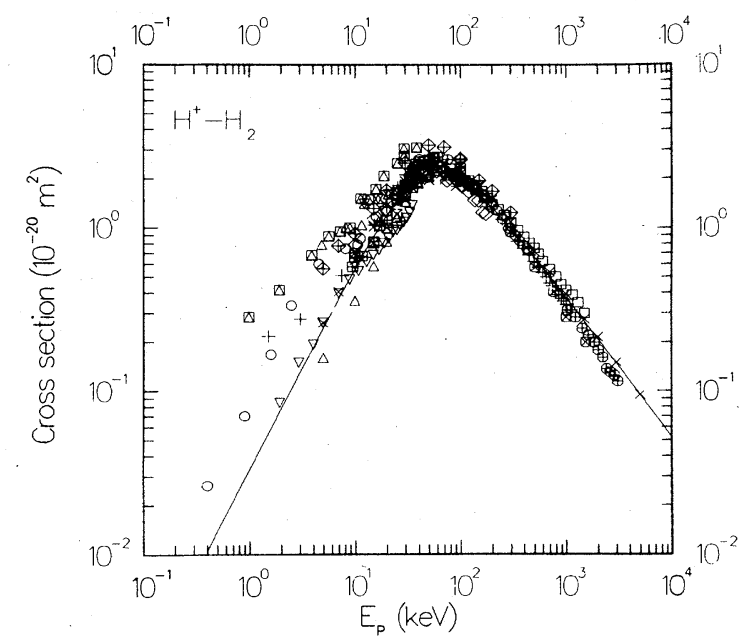

FIG. 13. Cross sections for ejection of electrons in $\mathbf{H}^{+}+\mathbf{H}_{2}$ collisions. The solid curve is the recommended fit. Experimental data: $\nabla$, Keene (1949); \& , Fogel' et al. (1955); O, Gilbody and Hasted (1957) and Gilbody and Lee (1963); $\triangle$, Afrosimov et al. (1958a); $\boxplus$, Schwirzke (1960); + $\left(E_{p}>100 \mathrm{keV}\right)$, Hooper et al. (1961); $\diamond$, Solov'ev et al. (1962); $\bigoplus\left(E_{p} \leq 100 \mathrm{keV}\right)$, Kuyatt and Jorgenson (1963); $\oplus$, Rudd and Jorgenson (1963), Rudd et al. (1966), and Rudd (1979); $\triangle$, Gordeev and Panov (1964); $+\left(E_{p}<100 \mathrm{keV}\right)$, Hollricher (1965); $\bigotimes$, De Heer et al. (1966); $*$, Desesquelles et al. (1966); $\oplus\left(E_{p} \geq 1000 \mathrm{keV}\right)$, Pivovar and Levchenko (1967); $\triangle$, Afrosimov et al. (1969); $\bigotimes$, Toburen and Wilson (1972); $\square$, Shah and Gilbody (1982); $\times$, Rudd et al. (1983).

the proton-impact cross section for $E_{p}>2 \mathrm{MeV}(T>1$ $\mathrm{keV}$ ) is in fairly good agreement with the high-energy behavior of electron-impact cross sections, although the slopes are somewhat different.

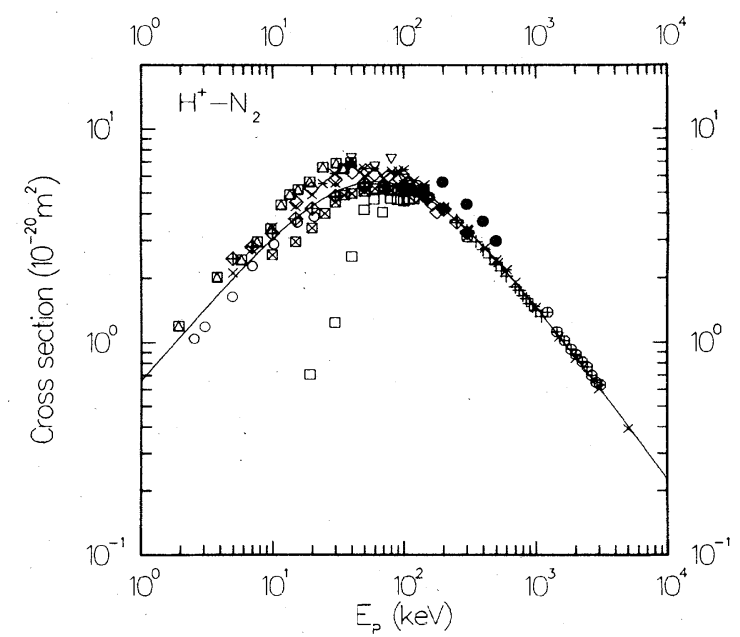

FIG. 14. Cross sections for ejection of electrons in $\mathrm{H}^{+}+\mathrm{N}_{2}$ collisions. The solid curve is the recommended fit. Experimental data: +, Hooper et al. (1961); $\diamond$, Solov'ev et al. (1962); \, Gordeev and Panov (1964); $\bigotimes$, De Heer et al. (1966); $*$, Dufay et al. (1966); $\bigoplus$, Pivovar and Levchenko (1967); $\nabla$, Collins and Kebarle (1967); O, McNeal and Clark (1969); $\square$, Schowengerdt and Park (1970); $\bullet$, Stolterfoht (1971b); $\oplus$, Crooks and Rudd (1971) and Rudd (1979); $\bigotimes$, Toburen and Wilson (1975); $X$, Rudd et al. (1983).

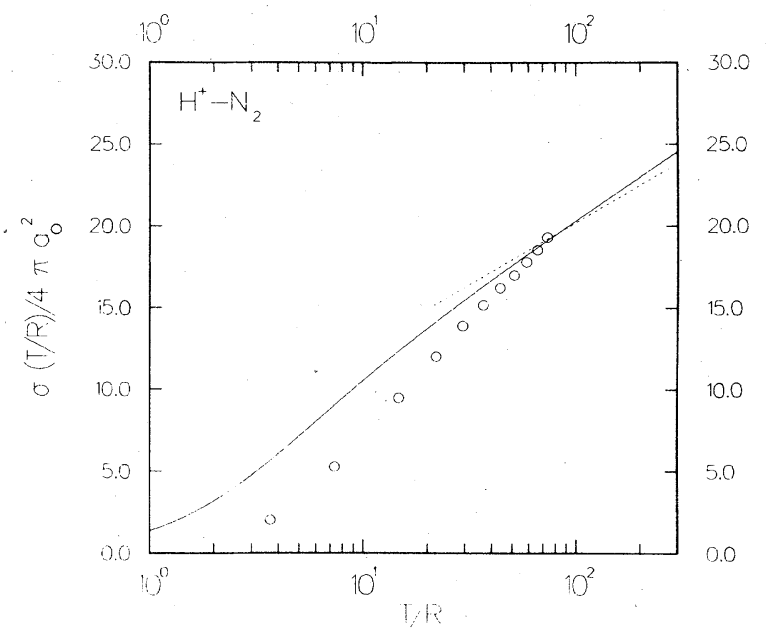

FIG. 15. Fano plot for ionization of $\mathrm{N}_{2}$. The solid curve is the recommended fit and the dashed line is the electron-impact ionization cross section recommended by Kim (1975). The circles represent electron-impact data measured by Rapp and Englander-Golden (1965).

\section{Oxygen}

The fitted value of $A$ is only about $6 \%$ higher than the optical value, so no adjustment has been made. The results are shown in Fig. 16.

\section{Carbon monoxide}

The value of $A$ from the initial fitting was 4.63, which is in poor agreement with the optical value of 3.67. But when $A$ was adjusted to the optical value and the other parameters recalculated for best fit, the agreement was almost equally good over most of the range and better at

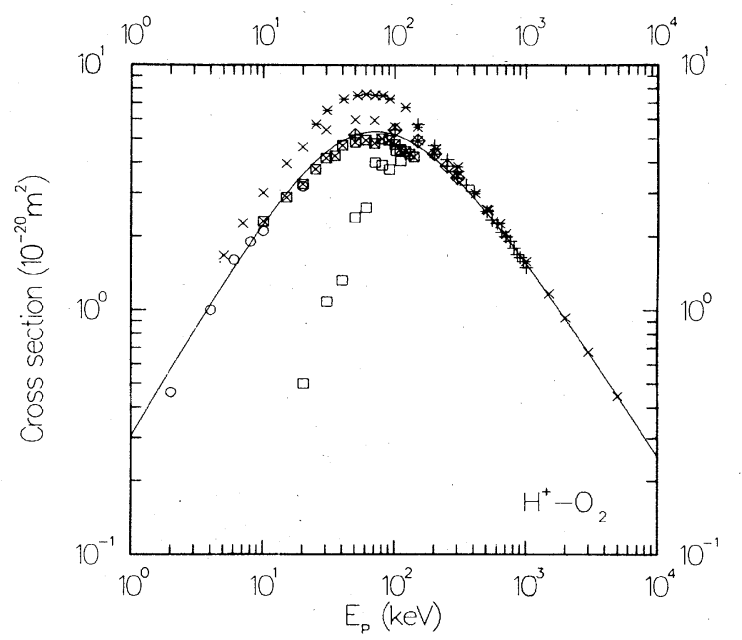

FIG. 16. Cross sections for ejection of electrons in $\mathrm{H}^{+}+\mathrm{O}_{2}$ collisions. The solid curve is the recommended fit. Experimental data: +, Hooper et al. (1961); $\bigotimes$, De Heer et al. (1966); $*$, Dufay et al. (1966); $\square$, Park et al. (1971); $\oplus$, Crooks and Rudd (1971); O, McNeal and Birely (1973); $\times$, Rudd et al. (1983). 


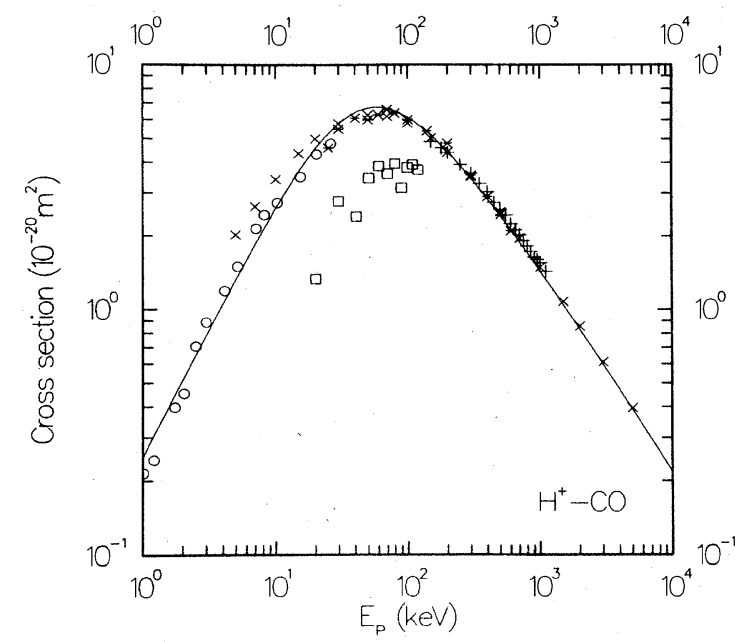

FIG. 17. Cross sections for ejection of electrons in $\mathrm{H}^{+}+\mathrm{CO}$ collisions. The solid curve is the recommended fit. Experimental data: +, Hooper et al. (1961); $*$, Poulizac et al. (1967); $\square$, Park et al. (1970); O, McNeal (1970); $\times$, Rudd et al. (1983).

the very highest energies. Therefore this set of parameters was retained. The results are shown in Fig. 17.

\section{Ammonia}

The fitted value of $A$ is only $7 \%$ above the optical value, so no adjustment was made. The small amount of data available appear to be reliable, although additional data are needed. See Fig. 18.

\section{Carbon dioxide}

Although the value of $A$ from the weighted fit (6.55) was $21 \%$ greater than the optical value, no adjustment was made in the fitted values, since the fit was very good

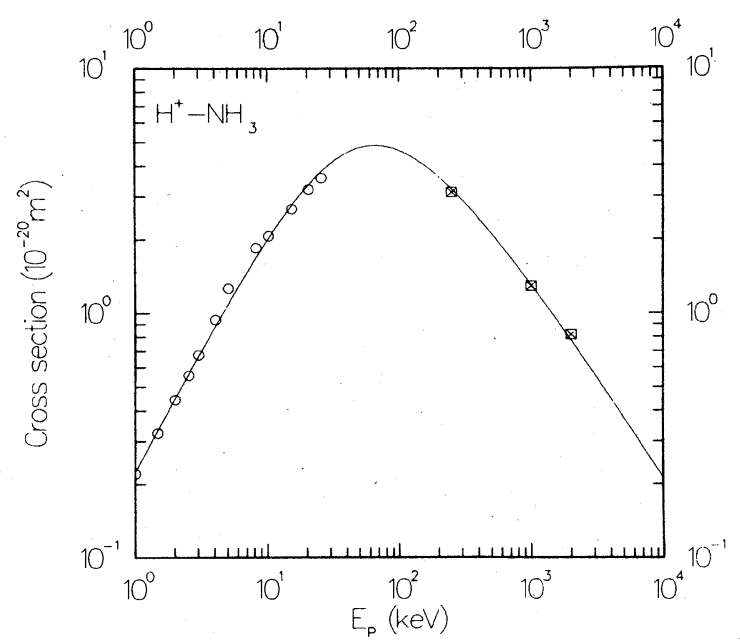

FIG. 18. Cross sections for ejection of electrons in $\mathbf{H}^{+}+\mathrm{NH}_{3}$ collisions. The solid curve is the recommended fit. Experimental data: O, McNeal (1970); $\otimes$, Lynch et al. (1976).

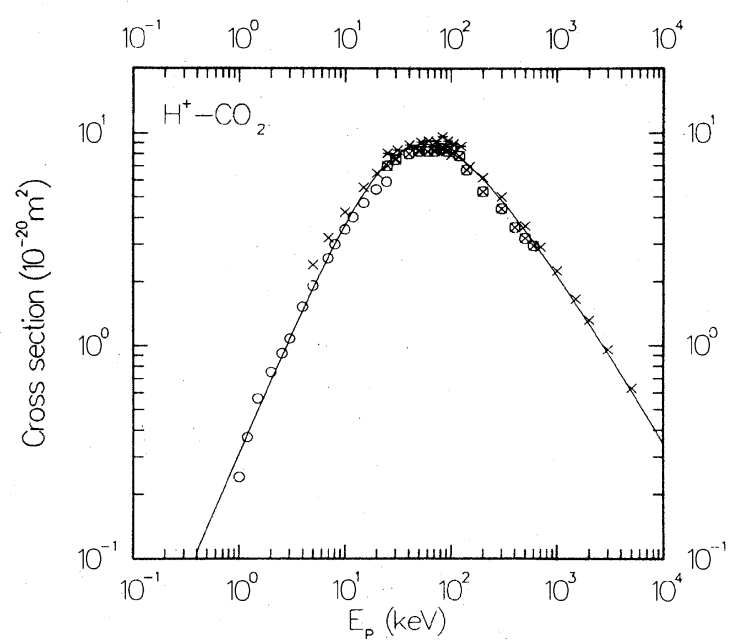

FIG. 19. Cross sections for ejection of electrons in $\mathrm{H}^{+}+\mathrm{CO}_{2}$ collisions. The solid curve is the recommended fit. Experimental data: $*$, Desesquelles et al. (1966); $\bigcirc$, McNeal (1970); $\bigotimes$, Poulizac and Dufay (1967); $\times$, Rudd et al. (1983).

and there was little spread in the data. Dissociative ionization may account for some of this discrepancy, but its magnitude is unknown. The results are shown in Fig. 19.

\section{Methane}

The results are shown in Fig. 20. The value of $A$ was adjusted slightly to the optical value of 4.55 . There is a 10-35\% discrepancy between the data of Lynch et al. (1976) and the data of Rudd et al. (1983) at higher energies, which causes the overall results to be somewhat uncertain. The data sets of McNeal (1970) and Rudd et al. (1983) were given the most weight.

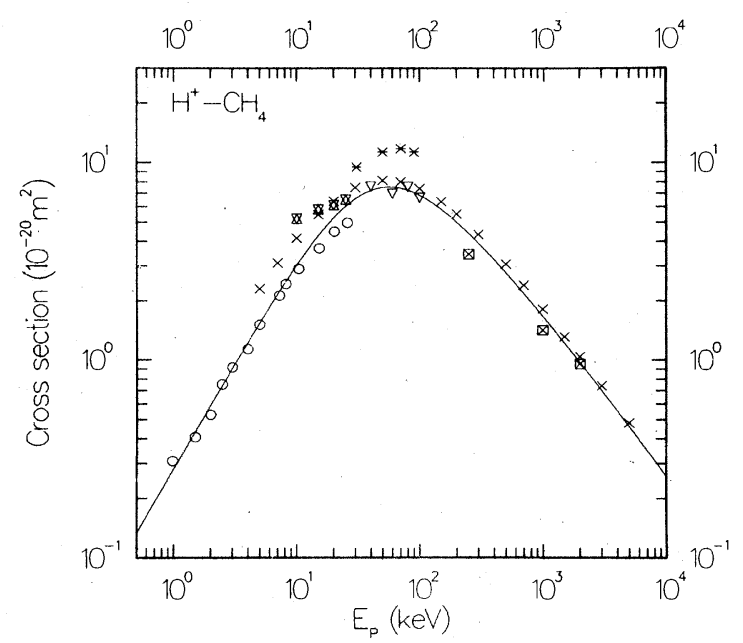

FIG. 20. Cross sections for ejection of electrons in $\mathbf{H}^{+}+\mathrm{CH}_{4}$ collisions. The solid curve is the recommended fit. Experimental data points: $*$, Desesquelles et al. (1966); $\nabla$, Collins and Kebarle (1967); O, McNeal (1970); $\bigotimes$, Lynch et al. (1976); $\mathbb{Z}$, Mach et al. (1977); $\times$, Rudd et al. (1983). 


\section{Other targets}

None of the other targets (see Table II) had data over a wide enough energy range to make a reliable fit possible.

\section{RECOMMENDATIONS FOR FUTURE WORK}

Although the first proton ionization measurement was made only 36 years ago, reasonably complete data now exist on most of the common gases. For atomic hydrogen, helium, neon, nitrogen, oxygen, carbon monoxide, and carbon dioxide, the data available allow us to make recommendations on the values of the high-energy cross sections that we believe are reliable to within $10 \%$.

The situation at intermediate and low energies is less satisfactory and becomes worse the lower the energy. Only in a few cases are the cross sections in the (1-50)$\mathrm{keV}$ region known to better than $35 \%$. Additional data are needed for atomic hydrogen below $40 \mathrm{keV}$, for xenon and ammonia at all energies, and for most of the target gases below $5 \mathrm{keV}$. A few highly accurate benchmarktype measurements for each gas at the lower energies would be very desirable. Because of the problems of beam deflection, beam neutralization, and small cross sections at low energies, a new approach is probably needed to achieve the desired accuracy. The method of Latypov and Shaporenko (1972) is a promising beginning in this direction. The only data on water vapor (Rudd et al., 1985) were published too recently to be included in this review.

For making accurate corrections to cross-section measurements, it would also be helpful to have certain auxiliary data. More extensive data on secondary-electron coefficients for slow ions on various kinds of metal surfaces are needed, as well as data on reflection of fast ions and sputtering.

Differences in multiple ionization between proton, electron, and photon impact must be taken into account in comparing these cross sections, especially for the heavier targets. Unfortunately, little data are available and theoretical methods for treatment of multiple ionization are not well developed. Additional work in this area is badly needed.

For the case of heavy atoms or molecules, some obvious effects need to be theoretically examined. It is known, for example, that the quality of the atomic wave function can significantly affect the results of a cross-section calculation. In this work, Hartree-Fock wave functions which are adequate for light atoms have been used. For heavy atoms, electron correlations and relativistic effects will be important and should be carefully examined.

Another problem that needs to be examined is the nonorthogonality of the continuum and bound-state wave functions. For $p$ states, the overlap integrals between the bound and continuum wave functions are substantial ( $>0.1$ ), and if no corrections are made, the resulting cross sections are unrealistically large. This problem is particularly severe in any calculations based on the plane-wave Born approximation. For this work, corrections were made by subtracting overlap integrals from affected matrix elements. However, this problem should be investigated more carefully using better orthogonalization methods.

Another major theoretical challenge lies in the area of low incident energies. In this energy region, the present theoretical results are not reliable. For slow protons $\left(E_{p} \lesssim 100 \mathrm{keV}\right)$, theory should use a molecular description of the entire colliding system (i.e., the incident proton, ejected electrons, and the residual ion), since these particles interact strongly. The distorted-wave Born approximation and any similar theories which treat the interaction between the proton and the target as a first-order perturbation are not adequate in this energy range. For instance, it is well known that charge transfer is important and should be considered for slow protons. A proper theory should treat direct ionization and charge transfer on an equal footing by coupling all colliding particles together. Two- and three-center approaches (e.g., SethuRaman et al., 1973; Winter and Lin, 1984), in which the colliding particles are described as a diatomic molecule, embody a proper physical picture, but these become numerically intractable for the proton energies of interest here, viz., 10-100 keV. Multiple ionization with subsequent autoionization is also an important, unsolved problem.

Finally, theoretical methods for the ionization of molecules need to be developed. The distorted-wave Born approximation used here gives reliable total cross sections for light atoms and high energies. This type of theory should also be reliable in the same energy range for small molecules, but no theoretical work to confirm this assumption has been reported.

As is evident from this review, experiment is far ahead of theory in most cases. Major systematic theoretical efforts will be required to change this situation.

\section{ACKNOWLEDGMENTS}

This work was supported by the Office of Standard Reference Data, National Bureau of Standards. We thank the National Science Foundation for support, one of us (M.E.R.) under Grant No. PHY80-25599 and another (D.H.M.) under Grant No. PHY83-10644. One of us (Y.K.K.) would like to acknowledge the support by the Department of Energy in the early stage of this study. Special thanks go to Patricia Ruttenberg for her significant contribution in testing the fitting formulas and determining the fitting parameters. The authors are especially grateful to Martin Berger, John Cooper, Gordon Dunn, and Thomas M. Miller for reading the manuscript and making helpful suggestions.

\section{REFERENCES}

Afrosimov, V. V., R. N. Il'in, and N. V. Fedorenko, 1958a, Zh. Eksp. Teor. Fiz. 34, 1398 [Sov. Phys.-JETP 7, 968 (1958)].

Afrosimov, V. V., R. N. Il'in, and N. V. Fedorenko, 1958b, Zh.

Tekh. Fiz. 28, 2266 [Sov. Phys.—Tech. Phys. 3, 2080 (1958)]. 
Afrosimov, V. V., G. A. Leiko, Yu. A. Mamaev, and M. N. Panov, 1969, Zh. Eksp. Teor. Fiz. 56, 1204 [Sov. Phys.-JETP 29, 648 (1969)].

Afrosimov, V. V., Yu. A. Mamaev, M. N. Panov, and V. Uroshevich, 1967, Zh. Tekh. Fiz. 37, 717 [Sov. Phys.-Tech. Phys. 12, 512 (1967)].

Barnett, C. F., and H. B. Gilbody, 1968, Methods of Experimental Physics, edited by B. Bederson and Wade L. Fite (Academic, New York), Vol. 7A, p. 390.

Barnett, C. F., J. A. Roy, E. Ricci, M. I. Wilher, E. W. McDaniel, E. W. Thomas, and H. B. Gilbody, 1977, Report No. ORNL-5206, Oak Ridge National Laboratory.

Becker, M., and A. Scharmann, 1969, Z. Naturforsch. A 24, 854.

Bederson, B., 1968, Methods Exp. Phys. A 7, 67.

Berkowitz, J., 1979, Photoabsorption, Photoioization and Photoelectron Spectroscopy (Academic, New York).

Bethe, H., 1930, Ann. Phys. (Leipzig) 5, 325.

Bevington, P. R., 1969, Data Reduction and Error Analysis for Physical Sciences (McGraw-Hill, New York).

Blaauw, H. J., R. W. Wagenaar, D. H. Barends, and F. J. De Heer, 1980, J. Phys. B 13, 359.

Burhop, E. H. S., and W. N. Asaad, 1972, Adv. At. Mol. Phys. $8,163$.

Collins, J. G., and P. Kebarle, 1967, J. Chem. Phys. 46, 1082.

Criswell, T. L., L. H. Toburen, and M. E. Rudd, 1977, Phys. Rev. A 16, 508.

Crooks, J. B., 1974, Ph.D. thesis (University of Nebraska).

Crooks, J. B., and M. E. Rudd, 1971, Phys. Rev. A 3, 1628.

De Heer, F. J., J. Schutten, and H. Moustafa, 1966, Physica (Utrecht) 32, 1766.

Desesquelles, J., G. D. Cao, and M. C. Dufay, 1966, C. R. Acad. Sci. Ser. B 262, 1329.

Douthat, D. A., 1979, J. Phys. B 12, 663.

Drawin, H. W., 1961, Z. Phys. 164, 513.

DuBois, R. D., 1984, Phy. Rev. Lett. 52, 2348.

DuBois, R. D., L. H. Toburen, and M. E. Rudd, 1984, Phys. Rev. A 29, 70.

DuBois, R. D., L. H. Toburen, and S. T. Manson, 1984, in $X$ Ray and Inner-Shell Processes in Atoms, Molecules, and Solids, edited by A. Meisel and J. Finster (Carl Marx University, Leipzig), p. 145.

Dufay, M., J. Desesquelles, M. Druetta, and M. Eidelsberg, 1966, Ann. Geophys. 22, 614.

Edwards, A. K., R. M. Wood, and M. F. Steuer, 1977, Phys. Rev. A 15, 48.

Eggarter, E., 1975, J. Chem. Phys. 62, 833.

Fedorenko, N. V., 1959, Usp. Fiz. Nauk. 68, 481 [Sov. Phys.Usp. 2, 526 (1959)].

Fedorenko, N. V., V. V. Afrosimov, R. N. Il'in, and E. S. Solovev, 1960, in Proceedings of the 4th International Conference on Ionization Phenomena in Gases, edited by N. R. Nillson (North-Holland, Amsterdam), Vol. I, p. 47.

Fite, W. L., R. F. Stebbings, D. G. Hummer, and R. T. Brackmann, 1960, Phys. Rev. 119, 663.

Fogel', I. M., L: I. Krupnik, and B. G. Safronov, 1955, Zh. Eksp. Teor. Fiz. 28, 589 [Sov. Phys.-JETP 1, 415 (1955)].

Fogel', I. M., R. P. Slabospitskii, and A. B. Rastrepin, 1960, Zh. Tekhn. Fiz. 30, 63 [Sov. Phys.-Tech. Phys. 5, 58 (1960)].

Froese-Fisher, C., 1972, Comput. Phys. Commun. 4, 107.

Gabler, H., 1974, Diplomarbeit thesis (Freie Universitat Berlin). See also Rudd et al. (1979).

Gaede, W., 1915, Ann. Phys. (Leipzig) 46, 357.

Ghosh, S. N., and W. F. Sheridan, 1957, J. Chem. Phys. 26,
480.

Gilbody, H. B., and J. B. Hasted, 1957, Proc. R. Soc. London Ser. A 240, 382.

Gilbody, H. B., and J. V. Ireland, 1963, Proc. R. Soc. London Ser. A 277, 137.

Gilbody, H. B., and A. R. Lee, 1963, Proc. R. Soc. London Ser. A 274, 365 .

Goldmann, F., 1932, Ann. Phys. (Leipzig) 10, 460.

Gordeev, Yu. S., and M. N. Panov, 1964, Zh. Tekh. Fiz. 34, 857

[Sov. Phys.-Tech. Phys. 9, 656 (1964)].

Hollricher, O., 1965, Z. Phys. 41, 187.

Hooper, J. W., 1961, Ph.D. thesis (Georgia Institute of Technology).

Hooper, J. W., E. W. McDaniel, D. W. Martin, and D. S. Harmer, 1961, Phys. Rev. 121, 1123.

Hvelplund, P., H. K. Haugen, and H. Knudsen, 1980, Phys. Rev. A 22, 1930.

Il'in, R. N., V. V. Afrosimov, and N. V. Fedorenko, 1959, Zh.

Eksp. Teor. Fiz. 36, 41 [Sov. Phys.-JETP 9, 29 (1959)].

Inokuti, M., 1971, Rev. Mod. Phys. 43, 297.

Ishii, H., and K. I. Nakayama, 1962, Transactions of the 8th National Vacuum Symposium (Pergamon, London), Vol. I, p. 519.

Keene, J. P., 1949, Philos. Mag. 40, 369.

Kim, Y.-K., 1975a, Radiat. Res. 64, 96.

Kim, Y.-K., 1975b, Radiat. Res. 64, 205.

Kim, Y.-K., and K. T. Cheng, 1978, Phys. Rev. A 18, 36.

Kim, Y.-K., and M. Inokuti, 1971, Phys. Rev. A 3, 665.

Klar, H., 1982, Z. Phys. A 307, 75.

Knudson, M., 1910, Ann. Phys. (Leipzig) 31, 205.

Kuyatt, C. E., and T. Jorgensen, Jr., 1963, Phys. Rev. 130, 1444.

Langenberg, A., and J. van Eck, 1976, J. Phys. B 9, 2421.

Latypov, Z. Z., and A. A. Shaporenko, 1972, Zh. Tekh. Fiz. 42, 151 [Sov. Phys.-Tech. Phys. 17, 117 (1972)].

Latypov, Z. Z., and A. A. Shaporenko, 1973, Pis'ma Zh. Eksp. Teor. Fiz. 18, 439 [JETP Lett. 18, 258 (1973)].

Levchenko, Yu. Z., L. I. Pivovar, and G. D. Tolstolutskaya, 1973, Zh. Eksp. Teor. Fiz. 64, 1991 [Sov. Phys.-JETP 37, 1004 (1973)].

Lynch, D. J., L. H. Toburen, and W. E. Wilson, 1976, J. Chem. Phys. 64, 2616.

Mach, R., H. Drost, H. Behlke, and H.-J. Spangenberg, 1977, Ann. Phys. (Leipzig) 34, 175.

Madison, D. H. 1973, Phys. Rev. A 8, 2449.

Madison, D. H., and S. T. Manson, 1979, Phys. Rev. A 20, 825.

Madison, D. H., and E. Merzbacher, 1975, in Atomic InnerShell Processes, edited by B. Crasemann (Academic, New York), Vol. 1, p. 1.

Manson, S. T., L. H. Toburen, D. H. Madison, and N. Stolterfoht, 1975, Phys. Rev. A 12, 60.

Massey, H. S. W., and E. H. S. Burhop, 1969, Electronic and Ionic Impact Phenomena (Oxford University, Oxford), Vol. I, Chap. 8.

Massey, H. S. W., and H. B. Gilbody, 1974, Electronic and Ionic Impact Phenomena (Oxford University, Oxford), Vol. IV, Chap. 23.

McCullough, R. W., and H. B. Gilbody, 1971, J. Phys. B 4, 1488.

McDaniel, E. W., J. W. Hooper, D. W. Martin, and D. S. Harmer, 1962, in Proceedings of the Fifth International Conference on Ionization Phenomena in Gases, edited by $\mathrm{H}$. Maecker (North-Holland, Amsterdam), Vol. I, p. 60. McNeal, R. J., 1970, J. Chem. Phys. 53, 4308. 
McNeal, R. J., and J. H. Birely, 1973, Rev. Geophys. Space Phys. 11, 633.

McNeal, R. J., and D. C. Clark, 1969, J. Geophys. Res. 74, 5065.

O'Hare, B. G., R. W. McCullough, and H. B. Gilbody, 1975, J. Phys. B 8, 2968.

Park, J. T., 1983, Adv. At. Mol. Phys. 19, 67.

Park, J. T., J. E. Aldag, J. M. George, J. L. Peacher, and J. H. McGuire, 1977, Phys. Rev. A 15, 508.

Park, J. T., J. M. George, J. L. Peacher, and J. E. Aldag, 1978, Phys. Rev. A 18, 48.

Park, J. T., D. R. Schoonover, and G. W. York, 1970, Phys. Rev. A 2, 2304.

Park, J. T., and F. D. Schowengerdt, 1969, Phys. Rev. 185, 152.

Park, J. T., F. D. Schowengerdt, and D. R. Schoonover, 1971, Phys. Rev. A 3, 679.

Pivovar, L. I., and Yu. Z. Levchenko, 1967, Zh. Eksp. Teor. Fiz. 52, 42 [Sov. Phys.-JETP 25, 27 (1967)].

Poulizac, M.-C., J. Desesquelles, and M. Dufay, 1966, C. R. Acad. Sci. Ser. B 263, 553.

Poulizac, M.-C., J. Desesquelles, and M. Dufay; 1967, Ann. Astrophys. 30, 301.

Poulizac, M.-C., and M. Dufay, 1967, Astrophys. Lett. 1, 17.

Puckett, L. J., and D. W. Martin, 1970, Phys. Rev. A 1, 1432.

Rapp, D., and P. Englander-Golden, 1965, J. Chem. Phys. 43 , 1464.

Rau, R., 1984, in Invited Papers of the XIIIth International Conference on the Physics of Electronic and Atomic Collisions, edited by J. Eichler, I. V. Hertel, and N. Stolterfoht (NorthHolland, Amsterdam), p. 711.

Rieke, F. F., and W. Prepejchal, 1972, Phys. Rev. A 6, 1507.

Rudd, M. E., 1977, reported in Criswell et al., 1977.

Rudd, M. E., 1979, Phys. Rev. A 20, 787.

Rudd, M. E., 1984, J. Quant. Spectrosc. Radiat. Transfer 31, 387.

Rudd, M. E., R. D. DuBois, L. H. Toburen, C. A. Ratcliffe, and T. V. Goffe, 1983, Phys. Rev. A 28, 3244.

Rudd, M. E., T. V. Goffe, R. D. DuBois, and L. H. Toburen, 1985, Phys. Rev. A 31, 492.

Rudd, M. E., and T. Jorgensen, Jr., 1963, Phys. Rev. 131, 666. Rudd, M. E., and J. H. Macek, 1972, Case Stud. At. Phys. 3, 47.

Rudd, M. E., and D. H. Madison, 1976, Phys. Rev. A 14, 128.

Rudd, M. E., C. A. Sautter, and C. L. Bailey, 1966, Phys. Rev. $151,20$.

Rudd, M. E., L. H. Toburen, and N. Stolterfoht, 1976, At. Data Nucl. Data Tables 18, 413.

Rudd, M. E., L. H. Toburen, and N. Stolterfoht, 1979, At. Data Nucl. Data Tables 23, 405.
Salin, A., 1972, Phys. B 5, 979.

Saxon, R. P., 1973, Phys. Rev. A 8, 839.

Schiff, L. I., 1949, Quantum Mechanics, 1st ed. (McGraw-Hill, New York), pp. 168-169.

Schowengerdt, F. D., and J. T. Park, 1970, Phys. Rev. A 1, 848.

Schram, B. L., A. J. H. Boerboom, W. Kleine, and J. Kistemaker, 1965, Physica 32, 749.

Schwirzke, F., 1960, Z. Phys. 157, 510.

SethuRaman, V., W. R. Thorson, and C. F. Lebeda, 1973, Phys.

Rev. A 8, 1316.

Shah, M. B., and H. B. Gilbody, 1981, J. Phys. B 14, 2361.

Shah, M. B., and H. B. Gilbody, 1982, J. Phys. B 15, 3441.

Smith, P. T., 1930, Phys. Rev. 36, 1293.

Solov'ev, E. S., R. N. Il'in, V. A. Oparin, and N. V. Fedorenko, 1962, Zh. Eksp. Teor. Fiz. 42, 659 [Sov. Phys.-JETP 15, 459 (1962)].

Steddeford, J. B., and J. B. Hasted, 1955, Proc. R. Soc. London 227, 466.

Stier, P. M., and C. F. Barnett, 1956, Phys. Rev. 103, 896.

Stolterfoht, N., 1971a, Z. Phys. 248, 81.

Stolterfoht, N., 1971b, Z. Phys. 248, 92.

Stolterfoht, N., 1975, reported in Manson et al., 1975, and Rudd et al., 1976.

Thomas, E. W., 1985, Atomic Data for Controlled Fusion Research Vol. III, "Particle Interaction with Surfaces," Oak Ridge National Laboratory Report No. ORNL-6088/V3.

Thomson, J. J., 1912, Philos. Mag. 23, 449.

Toburen, L. H., 1974, Phys. Rev. A 9, 2505.

Toburen, L. H., 1975, reported in Manson et al., 1975, and Rudd et al., 1976.

Toburen, L. H., S. T. Manson, and Y.-K. Kim, 1978, Phys. Rev. A 17, 148.

Toburen, L. H., and W. E. Wilson, 1972, Phys. Rev. A 5, 247.

Toburen, L. H., and W. E. Wilson, 1975, Rev. Sci. Instrum. 46, 851.

Toburen, L. H., W. E. Wilson, and L. E. Porter, 1977, J. Chem. Phys. 67, 4214.

Utterback, N. G., 1963, Phys. Rev. 129, 219.

Utterback, N. G., and T. Griffith, Jr., 1966, Rev. Sci. Instrum. $37,866$.

Utterback, N. G., and G. H. Miller, 1961, Phys. Rev. 124, 1477.

Wannier, G. H., 1953, Phys. Rev. 90, 817.

Wexler, S., 1964, J. Chem. Phys. 41, 1714; 44, 2221(E) (1966).

Williams, J. F., 1966, Phys. Rev. 150, 7.

Williams, J. F., and D. N. F. Dunbar, 1966, Phys. Rev. 149, 62.

Winter, T. G., and C. D. Lin, 1984a, Phys. Rev. A 29, 3071

Winter, T. G., and C. D. Lin, 1984b, Phys. Rev. A 30, 3323.

Younger, S., 1985, in Electron Impact Ionization, edited by T. D. Märk and G. H. Dunn (Springer, Vienna), pp. 1-23. 